\title{
Multi-Omics Reveals Microbial Roles and Metabolic Functions at the Spatiotemporal Niche in Pit Mud
}

\section{Dongliang Ren}

Jiangnan University

Shuangping Liu

Jiangnan University

\section{Suyi Zhang}

Sichaun Luzhou Laojiao Group Co. Ltd

Hui Qin

Sichuan Luzhou Laojiao Group Co. Ltd

Xiao Han

Jiangnan University

Jian Mao ( $\square$ maojian@jiangnan.edu.cn )

Jiangnan University https://orcid.org/0000-0002-3221-2492

\section{Research Article}

Keywords: Pit mud, Microbiota composition, Microbiota succession, Metabolites, Multi-omics analysis

Posted Date: February 11th, 2022

DOI: https://doi.org/10.21203/rs.3.rs-1251838/v1

License: (c) (i) This work is licensed under a Creative Commons Attribution 4.0 International License.

Read Full License 


\section{Abstract \\ Background}

Popular distilled Chinese strong-flavoured liquor (CSFL) is produced by solid fermentation in ground pits. Pit mud (PM), as a habitat for microbes, plays an important role in the production of CSFL. However, understanding the taxonomic composition, metabolic potential, and functional diversity of core microbes of PM in spatiotemporal niche remains a major challenge.

\section{Results}

Using a multi-omic approach of high-throughput full-length 16S rRNA, ITS sequencing, and metagenomics, we identified bacteria such as Caproiciproducens, Clostridium, Lactobacillus, Bellilinea, Petrimonas, Proteiniphilum, and Prevotella and the archaea Methanobrevibacter and Methanobacterium as the core microbiota in 30-, 100-, and 300-year-old cellars. Significant correlations between microbial communities and environmental factors showed that lactic, caproic, butyric, and acetic acids were the core driving forces for microbiota succession in different spatial locations and were mainly correlated with Caproiciproducens, Clostridium, Lactobacillus, Methanobrevibacter, and Methanobacterium. A total of 982 metabolites were detected using GC/MS and LC/MS, mainly including amino acids, peptides, and fatty acids, and correlations were shown between seven microorganisms and 12 amino acids and fatty acid metabolites. The crucial genes of flavour-relevant and substrate degradation pathways mainly included nine microorganism orders, with the most important being Clostridiales, Bacteroidales, and Methanobacteriales. However, only Clostridiales and Bacillales were potentially involved in alcoholaldehyde-carboxylate-ester metabolism.

\section{Conclusions}

The 30-, 100-, and 300-year-old cellars provided an ideal opportunity to understand the effect of cellar age on the microbial composition and functional diversity of the microorganisms in PM. Our detailed metagenomic analyses across the continuously used cells extend the known diversity of microorganisms involved in flavour generation and substrate degradation over a wide range of environmental conditions. The results indicate that Clostridiales and Bacteroidales are major microbiota orders for flavour generation and substrate degradation pathways, while archaea also play an important role in cooperation with bacteria in various flavour generation pathways. This study helps elucidate the core microbiota composition, different metabolic roles of microorganisms, and the formation mechanism of PM partial functions, thus providing a basic theory to support the regulation of Baijiu production.

\section{Background}


Numerous fermented foods, such as cheese, wine, bread, and yoghurt, have been consumed for years. These fermented foods are produced with complex microbiota from raw materials and the environment ${ }^{[1]}$. Chinese liquors are traditional fermented distilled spirits and are classified into four basic aroma types: strong, light, soy sauce, and rice aroma types. Chinese strong-flavoured liquor (CSFL), also called 'Luzhou-flavour liquor', is famous for its distinctive flavour and unique liquor culture and accounts for more than $70 \%$ of Chinese liquor production ${ }^{[2,3]}$. CSFL production is a typical recycling process that uses solid-state fermentation (Fig. 1). In brief, raw material mixtures are anaerobically fermented for 45-90 days or longer in a specialised rectangular soil pit (Fig. 1) lined with pit mud (PM) ${ }^{[4]}$. Fermentation involves numerous microbes originating from the PM and Daqu ${ }^{[1]}$. PM, a fermented clay, is considered a small-scale micro-ecosystem that contains various microbes, contributing to the simultaneous saccharification and alcoholic fermentation of raw materials. Microbes in the PM can produce complex and diverse metabolites such as small-molecule organic acids (e.g., caproic, butyric, acetic, and lactic acids), which are the key precursors of flavour esters, to largely determine the style and quality of CSFL ${ }^{[2}$, 5-8]. Many studies have elaborated that the class Clostridia such as Clostridial cluster IV and Clostridium are crucial microbes in PM and are responsible for the formation of various aromatic compounds (e.g., fatty acids, alcohols, and phenols) in CSFL ${ }^{[9-11]}$. Clostridial microbes can utilise ethanol, lactic acid, and acetic acid as substrates to synthesise hexanoic and butyric acids ${ }^{[12,13]}$. Lactobacillus is the dominant genus responsible for ethanol oxidation and lactate production ${ }^{[14]}$. With the degradation of starch and other macromolecules during fermentation, Huangshui, a leaching solution of fermented grains, accumulates with CSFL fermentation as by-product, which contains a variety of microbial metabolites such as lactic acid, ethanol, and acetic acid ${ }^{[15]}$, which can be further metabolised by PM microbiota. In general, CSFL quality also improves with increasing cellar age; high-quality liquor is produced only in old cellars, which are maintained for at least 20 years by continuous use ${ }^{[16]}$. In particular, some cellars have been used for several hundred years without interruption: for example Wuliangye, Jiannanchun, and Luzhou Laojiao are brewed in such long-aged cellars ${ }^{[17,18]}$, and PM with high quality has a stable and robust microbial structure ${ }^{[19]}$.

Recently, great progress has been made in investigating the microbial composition of PM. Taxonomic studies using culture- and molecular-based (such as sequencing and profiling of 16S rRNA genes and metagenomics) methods have shown that bacteria and archaea are core populations in the PM microbial community. The dominant prokaryotic phyla mainly consists of bacterial phyla such as Firmicutes, Bacteroidetes, Chloroflexi, Actinobacteria, Synergistetes, Proteobacteria, and other unclassified bacteria, which include Clostridium, Caproiciproducens, Ruminococcus, Caloramator, Sedimentibacter, Syntrophomonas, Lactobacillus, and Petrimonas genera, and archeal phylum Euryarchaeota that includes Methanobrevibacter, Methanobacterium, and Methanoculleus genera ${ }^{[14,20]}$. It is noteworthy that Firmicutes predominate in many PM microbial communities ${ }^{[21,22]}$. The genome approach is a referencefree assembly that provides a powerful tool for understanding the metabolic diversity of microbiomes and improves the taxonomic classification of microbiome populations ${ }^{[23,24]}$. Subtle relationships between the environment and microbial species can affect the microbial community structure ${ }^{[25]}$, and the 
moisture content in the pit can directly affect the $\mathrm{pH}$, humus, and growth of the microbial flora. The proper $\mathrm{pH}$ of PM not only promotes CSFL fermentation but also facilitates the formation of aroma precursor substances and improves the quality of the original liquor. Microorganisms are continuously enriched, and additional populations are derived during the long-term reciprocating fermentation process. In fact, the interaction between these microorganisms and physicochemical indices has formed a complex microbial ecosystem ${ }^{[26]}$.

Although many studies have elaborated the microbiota composition and the factors driving the microbiota succession in PM, previous studies have only focused on the microbiota composition in the whole cell without considering the spatial locations. Moreover, only limited knowledge of the microbial composition in different spatial locations of PM is available, and the factors that drive the microbiota succession in PM with hundreds of year intervals need to be clarified. Concurrently, little is known about the metabolic potential and functional properties of the PM microbiota owing to the lack of knowledge of complex microbiota interactions. Therefore, understanding the relationship among microbes, metabolites, and functional enzymes in PM remains a major challenge.

To address some of these problems, using a multi-omic approach, this study aimed to (1) characterise the microbiota community composition, analyse the driving factors for microbial succession, and reveal the relationships between chemical properties and microbial community in different PMs; and (2) investigate the differential metabolites and functional enzymes produced by microorganisms related to flavour generation and substrate degradation pathways.

\section{Results}

\section{Analysis of PM physicochemical factors}

The levels of PM moisture, TN, AP, pH, and acetic, caproic, butyric, and lactic acid content from different cellar samples changed significantly with increasing age of the cellar (Table 1, Fig. 2). pH is an important factor that has a cumulative effect on the properties of PM; however, it did not show differences in different spatial locations in the 30-, 100-, and 300-year-old cellars ( $p>0.05)$. The relative moisture content showed a significant difference in the 100- and 300-year-old cellars. The moisture content in position 4 was significantly lower than that in positions 1 and $2(p<0.05)$. For the same positions, the moisture content in the 300-year-old cellars was slightly higher than that in the 100-and 30-year-old cellars, but the difference was not significant ( $p>0.05$ ). The moisture content produced by microbial metabolism gradually accumulated and sank to the bottom of the cellar during fermentation, resulting in higher moisture content at the bottom of the cellar compared with that at other spatial positions. Nutrients are also important contributors to the biochemical properties of PM. The concentration of TN at position 4 was significantly lower than that at other positions in the 30-, 100-, and 300-year-old cellars $(p<0.05)$. Notably, the concentration of TN at position 1 in the 300-year-old cellar was significantly lower than that in the 30- and 100-year-old cellars. The concentration of AP at position 1 in the 100- and 300-year-old 
cellars was significantly higher than that at positions 2,3 , and $4(p<0.05)$ and at positions 2 and 4 , respectively.

Organic acids are important precursors of esters in CSFL and inevitably affect the quality of CSFL. We compared the organic acid contents spatiotemporally in the PM. The acetic acid content (Fig. 2A) was significantly higher at position $3(p<0.05)$ and position $1(p<0.05)$ than that at other positions in the 30 and 100-year-old cellars and 300-year cellars, respectively. The butyric acid content (Fig. 2B) showed no significant spatial difference in the 30-year-old cells $(p>0.05)$ but was significantly higher at position 1 than that at other spatial locations $(p<0.05)$ in the 100- and 300-year-old cellars. The caproic acid (CA) content (Fig. 2C) showed different trends in the different cellar types. The CA content at positions 2 and 1 was significantly higher than that at other positions $(p<0.05)$ in the 30 - and 300 -year-old cellars, respectively. As for lactic acid content (Fig. 2D), positions 4 and 1 showed significantly higher levels than other spatial locations in the 30- $(p>0.05)$ and in the 100- and 300-year-old cellars $(p<0.05)$, respectively. In summary, organic acids accumulated at the bottom of the pit with continuous CSFL fermentation, leading to a high content of organic acid at the bottom of the pit, when compared with other spatial locations. In addition, the total organic acid content increased with the continuous use of pits.

\section{Microbial succession in different cellars}

\section{Spatiotemporal differences of microbial diversity in PM}

Succession patterns of PM microbial communities in 30-, 100-, and 300-year-old cellars were investigated. High-throughput sequencing was applied to obtain information on the active microbial community in the three cellar types at four positions, and sequence similarity thresholds were used to define the OTUs. Based on the sequencing results, the total reads were correlated with bacterial and archaeal phyla. The Good's coverage estimator revealed that $91.72-94.05 \%$ of bacterial OTUs were obtained in all PM samples, which indicates that the generated data were reasonable reflection of the microbiota community. In addition, the bacterial Shannon index with a range of $5.41 \pm 0.96$ to $5.48 \pm 0.86$ and the bacterial Simpson index with a range of $0.9 \pm 0.07$ to $0.95 \pm 0.02$ at different PM samples were obtained, and there were significant differences $(p<0.05)$ among the different PM samples (Table 1$)$, suggesting similar overall species diversity.

For bacterial community composition, only prokaryotic abundance differences were found, without prokaryotic structural differences. At the phylum level, Firmicutes, Bacteroidetes, Euryarchaeota, Actinobacterium, Chloroflexi, and Proteobacteria were predominant in the 30-, 100-, and 300-year-old PM samples. At the genus level, 221 genera were detected. Bacterial genera such as Caproiciproducens, Clostridium, Lactobacillus, Bacillus, Weissella, Sedimentibacter, Atopobium, Brachybacterium, Ralstonia, Acinetobacter, Proteiniphilum, Anaerocella, Prevotella, Bellilinea, Petrimonas, Staphylococcus, Caloramator, Lysinibacillus, Caldicoprobacter, Caloribacterium and archeal genera such as Methanobacterium and Methanobrevibacter were detected with relative abundances higher than $0.1 \%$ in the 30-, 100-, and 300-year-old PM samples (Fig. 3). In this study, we defined core microbiota genera as 
those detected in all PM samples and with relative abundance higher than $1.0 \%$ in all samples. Based on this, the core microbiota genera were Lactobacillus, Caproiciproducens, Clostridium, Proteiniphilum,

Prevotella, Bellilinea, Petrimonas, Methanobacterium, and Methanobrevibacter and accounted for $60.28 \%$, $84.39 \%$, and $80.65 \%$ in the $30-, 100$ - and 300 -year-old PM samples, respectively. Notably, Prevotella, Bellilinea, Petrimonas, Methanobacterium, and Methanobrevibacterwere increased in the 100- and 300year-old PM samples when compared with those in the 30-year-old PM samples, especially for Methanobacterium and Methanobrevibacter. We further investigated the differential microbes among the three PM samples. The results (Fig. 3D) indicated that Proteiniphilum, Caproiciproducens,

Methanobacterium, and Methanobrevibacter were the main differential microorganisms among the 30-, $100-$, and 300-year-old samples. With the continuous use of the cellars, microbes will inevitably undergo succession progress. We calculated the beta nearest taxonomic index ( $\beta N T I)$ value to compare the influence of the two (deterministic and stochastic) assembly processes of the 30-, 100-, and 300-yearold cellars (Fig. 3E) to compare and analyse the differences in the construction of microbial communities in the different PM cellars. The results showed that both deterministic and stochastic processes promoted the assembly of the microbiome in the PM cellars, with the pit used for a long time, especially for 300-year-old cellars, the microbial composition follows a deterministic process.

\section{Microbial diversity in different spatial locations}

The cellar environment changed with the CSFL fermentation process, such as the Huangshui generation. It gradually changed from a micro-anaerobic to an anaerobic environment, leading to a diversity of microbial communities in different spatial locations. At position 1 (Additional file: Figure S1A), Lactobacillus, Bellilinea, Methanobrevibacter, Petrimonas, and Prevotella were found in the 30-, 100-, and 300-year-old cellars. Petrimonas (13.18\%) showed a higher relative abundance in the 100-yearold PM samples than that in the 30- and 300-year-old PM samples. Moreover, Lactobacillus (41.82\%) and Prevotella (1.78\%) were higher in the 300-year-old PM samples than in the 30 - and 100-year-old PM samples, but the relative abundance of Bellilinea $(0.7 \%)$ for the 300 -year cellar was lower than that for the 30- and 100-year samples. In contrast, Bacillus, Weissella, Brachybacterium, Staphylococcus, Anaerocella, Caloramator, Caldicoprobacter, and Caloribacterium were only detected in the 30-year, 100-year, and 300year-old PM samples, respectively.

At position 2 (Additional file: Figure S1-B), the dominant microbes were Lactobacillus, Bellilinea, Methanobrevibacter, Methanobacterium, Petrimonas, Caproiciproducens, and Prevotella. The Prevotella (12.38\%) and Lactobacillus (12.34\%) in the 30-year-old samples were higher than in the 100- and 300year-old PM samples. Bellilinea (18.04\%), Petrimonas (21.18\%), and Methanobrevibacter (30.16\%) in the 300 -year-old samples were higher than in the 30 - and 100-year-old samples, and Methanobacterium (9.96\%) and Caproiciproducens (15.63\%) in the 100-year-old samples were higher than in the 30- and 300year-old samples. Anaerocella and Lysinbacillus were only detected in the 30- and 300-year-old samples, respectively. 
At position 3 (Additional file: Figure S1-C), the core microbes were Bellilinea, Methanobrevibacter, Methanobacterium, Petrimonas, Proteiniphilum, Clostridium, and Prevotella. Prevotella $(26.45 \%)$ and Methanobacterium (9.28\%) in the 30-year-old samples were higher than in the 100- and 300-year-old samples, whereas Proteiniphilum (14.27\%), Bellilinea (29.09\%), and Clostridium (6.68\%) in the 300-yearold samples were higher than in the 30 - and 100 -year-old samples. Methanobrevibacter $(41.79 \%)$ in the 100-year-old PM samples was higher than in the 30- and 300-year-old PM samples.

At position 4 (Additional file: Figure S1-D), the core microbes were Bellilinea, Methanobrevibacter, Petrimonas, Lactobacillus, and Prevotella. Petrimonas (19.27\%) and Prevotella (14.53\%) in the 30-yearold PM samples were higher than in the 100- and 300-year-old PM samples, and Methanobrevibacter (52.33\%) and Lactobacillus (21.78\%) in the 100-year-old samples were higher than in the 30- and 300year-old samples. Moreover, Bellilinea in the 300-year-old PM samples was higher than in the 30-and 100year-old PM samples.

Differences in the physicochemical properties of different spatial positions in the pit, such as oxygen, moisture content, nutrients, and the contact between the jiupei (fermented grains) and PM, will inevitably lead to differences in microbial composition and abundance. Notably, the relative abundance of the same microorganism is also different in the use of the same year pit. In summary, the microbiota composition among the four spatial locations were different; for example, Caproiciproducens and Lactobacillus were concentrated at the bottom of the cellar, while Proteiniphilum and Clostridium were highly abundant at positions 3 and 4 . In addition, some genera were only detected in specific spatial locations, indicating the complex interactions between the microorganisms. Moreover, the microbial composition also correlated with the physicochemical environment in the cellars, and the diversity of microbes could influence the flavour substances in CSFL.

After studying the microbial communities of different PM samples, we further evaluated the similarities and differences in the microbiota using a Bray-Curtis approach. A non-metric multi-dimensional scaling analysis revealed that all samples in bacteria were clustered into two parts and showed year-dependent clustering (Fig. 4A). In summary, samples from the 30-, 100-, and 300-year-old samples were centralised and formed clusters, especially for 30 - and 100-year-old samples, suggesting that samples from different years are possibly in different transitional states and bacterial communities in the 30- and 100-yearold cellars had similar microbial compositions. The microbial community continues to evolve over time, stabilises, and gradually forms a stable microbial environment. Therefore, the age of the cellar is the main factor driving microbial succession.

\section{Relationships of microbial community with physicochemical properties}

Environmental factors in fermentation can affect the growth and metabolism of microorganisms. Hence, physicochemical factors $(\mathrm{pH}$, moisture content, TN, AP, acetate, caproate, butyrate, and lactate) were selected and analysed to reveal their connection with the microbiota community (Fig. 4B). The results indicate that the contents of lactate, butyrate, acetate, and caproate significantly affected microbial succession change $(p<0.05)$ and that lactate and caproate 
contents were positively correlated with prokaryotic communities in the 30-, 100-, and 300-yearold samples at positions 1 and 2. Moreover, butyrate and acetate contents were positively correlated with prokaryotic communities in the 30- and 100-year-old PM samples at position 2.

To clarify the relationships between specific genera and driving factors, we analysed their correlation using Spearman's coefficient $(\mathrm{p}<0.05)$. Organic acids significantly affected the core microbiota Lactobacillus, Caproiciproducens, Clostridium, Proteiniphilum, Prevotella, Bellilinea, Petrimonas, Methanobacterium, and Methanobrevibacter, while TN, AP, and moisture content affected a few families. Specifically, at position 1 (Additional file: Figure S2-A), lactic acid was positively correlated with levels of Caproiciproducens. In addition, butyric acid and acetic acid were positively correlated with Methanobacterium and Caproiciproducens, Caproic acid was negatively correlated with Petrimonas, and TN was negatively correlated with Prevotella and Lactobacillus. At position 2 (Additional file: Figure S2B), lactic acid was negatively correlated with Clostridium yet positively correlated with Lactobacillus, butyric acid was negatively correlated with Petrimonas yet positively correlated with Prevotella, acetic acid was positively correlated with Lactobacillus and Caproiciproducens, CA was negatively correlated with Petrimonas yet positively correlated with Lactobacillus, and moisture content was negatively correlated with Prevotella. At position 3 (Additional file: Figure S2-C), lactic acid and AP were the two important factors influencing microbial community composition. For example, lactic acid was positively correlated with Methanobrevibacter, but AP was negatively correlated with Ralstonia, Bellilinea, and Proteiniphilum and negatively correlated with Clostridium. At position 4 (Additional file: Figure S2-D), CA and butyric acid were negatively correlated with Methanobrevibacter, while butyric acid and lactic acid were positively correlated with Methanobacterium.

In summary, physicochemical factors such as TN, AP, and organic acids played an important role in determining the microbiota composition, and microbes in different spatial locations were affected by different factors, because the microbial composition differed spatially.

\section{Metabolomic analysis of PM}

In the untargeted metabolomics analysis, we identified 5991 metabolites, of which 714 were identified by gas chromatography (GC-MS), and 5277 were identified by liquid chromatography (LC-MS) from PM samples collected over different years that are continuously used. After filtering and quality control (deletion of unknown compounds), 982 metabolites were left for further analysis.

\section{Classification of total metabolites}

We first classified the metabolites detected by GC-MS and LC-MS. The results showed that amino acids, peptides, and analogues, fatty acids, and conjugates were the main metabolites and accounted for approximately half of the metabolites (Additional file: Figure S3). Metabolites from PM also contribute to the liquor flavour. Fatty acids, amino acids, and peptides are decisive compounds in CSFL because they can produce flavour compounds as precursors in CSFL production. 


\section{Analysis of differential metabolites}

We selected the metabolites according to the standard of $P$ value $<0.1$ and VIP value $>1$ to obtain the differential metabolites in different cell types.

In the position under the Huangshui fluid, there were 129 differential metabolites in the 30- and 100-yearold cellars (Additional file: Figure S4-A), of which 105 were up-regulated and 24 were down-regulated. There were 86 differential metabolites in the 30- and 300-year-old cellars (Additional file: Figure S4-B), of which 60 were up-regulated and 26 were down-regulated. Of 100 differential metabolites found in the 100- and 300-year-old cellars (Additional file: Figure S4-C), 41 were up-regulated and 59 were downregulated.

In the position of the Huangshui fluid, 68 differential metabolites were found in the 30- and 100-yearold PM samples (Additional file: Figure S4-D), of which 29 were up-regulated and 39 were down-regulated, and of the 247 differential metabolites in the 30- and 300-year-old cellars (Additional file: Figure S4-E), 95 were up-regulated and 152 were down-regulated. There were 178 differential metabolites in the 100-and 300-year-old cellars (Additional file: Figure S4-F), of which 77 were up-regulated and 101 were downregulated.

\section{Analysis of differential metabolic pathways between different cellars}

The main flavour compounds in CSFL are esters, alcohols, aldehydes, ketones, phenol, and pyrazine compounds. According to our results, differential metabolites were related to a variety of metabolic pathways. We selected differential metabolites for their potential participation in the substrate degradation and flavour development of PM. Dodecanoic, caprylic, myristic, arachidonic, $y$-linolenic, and eicosapentaenoic acids were related to the biosynthesis of fatty acids and unsaturated fatty acid pathways, while threonine, asparagine, phenylalanine, isoleucine, aspartic acid, valine, proline, and phenylpyruvic acid were related to the biosynthesis of amino acids. Malic acid, succinic acid, and $\gamma^{-}$ aminobutyric acid were related to the butanoate and pyruvate metabolism pathways. The relative concentrations of the metabolites were also compared. Under the Huangshui fluid (Table S1), compared to the 30-year-old samples, the compounds $\gamma$-aminobutyric acid, threonine, proline, phenylalanine, dodecanoic acid, succinic acid, and valine were increased in the 100-year-old PM samples and decreased in the 300-year-old PM samples. The concentrations of arachidonic acid, eicosapentaenoic acid, caprylic acid, malic acid, myristic acid, isoleucine, glyceric acid, and $\beta$-hydroxypyruvate in the 30-year-old PM samples were higher than in the 100-and 300-year-old PM samples. The concentrations of phenylpyruvic acid and $y$-linolenic acid in the 300-year-old samples were higher than in the 30- and 100-year-old PM samples. At the position of the Huangshui fluid (Table S2), compared to the 30-year-old samples, the compounds dodecanoic acid and a-linolenic acid were increased in the 100-year-old PM samples and decreased in the 300-year-old samples. The concentrations of homoserine, saccharopine, threonine, succinic acid, and aminoadipic acid in the 30-year-old PM samples were higher than in the 100- and 300year-old PM samples. The concentrations of $y$-linolenic acid, aspartic acid, caprylic acid, glucuronic acid, myristic acid, asparagine, and arachidonic acid in the 300-year-old PM samples were higher than in the 
30- and 100-year-old PM samples. Figure 6A shows the reciprocal connections between the differential metabolites and KEGG pathways.

We further analysed the relationship between core microbiota and PM metabolites (Additional file: Figure S5). The correlation coefficients indicated a strong relation $(0.6<\mid$ Spearman's $\mid<1)$. Seven of the nine main genera were involved in the distribution of certain metabolites. Positive correlations were observed between Caproiciproducens and isoleucine, gluconic lactone, and glyceric acid, Lactobacillus and CA, Methanobrevibacter and lactic acid, and Bellilinea and Proteiniphilum and caprylic acid.

\section{Metagenomic analysis}

\section{Metagenomic microbial community composition}

Among all sequences that passed the quality control, $62.15-64.1 \%, 21.81-27.92 \%$, and $0.01 \%$ were identified as fragments originating from bacteria, archaea, and eukaryotes, respectively. The dominant bacterial phyla were Firmicutes (19.84-35.71\%) and Bacteroidetes (8.11-19.57\%). The archaeal reads were mainly affiliated with Euryarchaeota (21.75-27.8\%). A small proportion of eukaryotic reads (less than $0.1 \%$ ) were detected in the PM samples. This indicated that the PM microbiota was dominated by prokaryotes (Additional file: Figure S6).

\section{Functional gene profiles of the PM metagenome}

To investigate the metabolic potential of the PM microbiome, microbial genes aligned against the KEGG database among all PM samples were categorised into three levels of pathways based on metagenomic sequencing data. The functional gene profile at level 2 was mainly composed of carbohydrate metabolism, amino acid metabolism, energy metabolism, and translation metabolism in PM samples (Additional file: Figure S6-C).

We selected 135 enzymes from the KEGG database for their potential participation in the substrate degradation and flavour development of PM, and they were then grouped into 20 functional assemblies. Starch and sucrose metabolism are the primary sources of all compounds in the process of fermentation. According to starch and sucrose metabolism, the key enzymes alpha-amylase (EC 3.2.1.1) and glucan 1,4-alpha-maltohydrolase (EC 3.2.1.133) can catalyse starch hydrolysis to dextrin and maltose. The major producers of these two enzymes were Clostridiales (mainly Clostridium), Anaerolineales (mainly Bellilinea), Methanomicrobiales (mainly Methanoculleus), and Methanosarcinales (mainly Methanosarcina). Glucose is generally generated from starch during fermentation and is converted to pyruvate through the activities of aldose 1-epimerase (EC 5.1.3.3) and glucokinase (EC 2.7.1.2), while aldose 1-epimerase catalyses the interconversion of $a$ - and $\beta$-terminal isomers of hexose, which are mainly produced by Petrimonas, Prevotella, and Clostridium. Moreover, glucokinase is related to the degradation of glucose and can catalyse glucose phosphorylation, which is mainly produced by Petrimonas, Clostridium, Prevotella, Methanoculleus, and Syntrophomonas. The above-mentioned 
microbiota genera were considered the major users of polysaccharides, and the generated glucose could be utilised by other taxa.

As for the flavour-generation metabolism pathways, CA is generally produced by anaerobic bacteria via the chain elongation pathway. The oxidation of ethanol can provide energy (acetyl-CoA) to sequentially elongate the carbon chain of carboxylic acids (acetic acid to n-butyric acid to CA).

Petrimonas, Methanoculleus, Syntrophomonas, and Methanobacterium were the most prevalent genera that produced the enzymes (EC 2.3.1.9, 1.3.1.9, 2.3.1.16, and 4.2.1.55) involved in CA synthesis. Moreover, Lactobacillus, Clostridium, and Methanoculleus were the predominant genera that produced lactic acid from pyruvate, mainly catalysed by L-lactate dehydrogenase (EC 1.1.1.27) and D-lactate dehydrogenase (EC 1.1.1.28). In addition, lactate dehydrogenase (EC 1.1.2.3, EC 1.1.2.4), which can consume lactic acid to produce pyruvate, reduces the production of lactic acid. Conversely, Sedimentibacter and Clostridium were the main potential groups converting lactate back to pyruvate via D-lactate dehydrogenase (EC 1.1.2.4), while only a few species were annotated to produce lactate dehydrogenase (EC 1.1.2.3).

Petrimonas was the predominant microbiota using pyruvate dehydrogenase (EC 1.2.4.1 and EC 2.3.1.12), which oxidised pyruvate to generate acetyl-CoA. This is a critical link in multiple metabolic pathways, contributing to aromas in CSFL, and can be oxidised to alcohol via acetyl-CoA synthetases (EC 6.2.1.13), aldehyde dehydrogenase (EC 1.2.1.3), and alcohol dehydrogenase (EC 1.1.1.1, EC 1.1.1.2). Among these, alcohol dehydrogenase is one of the most important enzymes involved in ester substance generation according to the alcohol-aldehyde-carboxylate-ester metabolism pathway. The gene coding for alcohol dehydrogenase was mainly enriched in microbes belonging to Clostridium. Butyrate is another basic aroma in CSFL and is produced from butyryl-CoA by the catalytic activities of acetate CoA transferase (EC 2.8.3.8) or butyrate kinase (EC 2.7.2.7). The genus source of butyrate kinase (EC 2.7.2.7) was Caloramator, Petrimonas, and Sedimentibacter, while the genus source of acetate CoA-transferase (EC 2.8.3.8) was Petrimonas, Sedimentibacter, and Proteiniphilum. Genes of various pathways to form acetate were detected in the microbiota, with acetyl-CoA and acetyl-P as intermediates. The key enzymes aldehyde dehydrogenase (EC 1.2.1.3), acetate kinase (EC 2.7.2.1), acetate-CoA ligase (EC 6.2.1.13), and pyruvate dehydrogenase (EC 1.2.5.1) involved in acetate metabolism were mainly produced by Clostridiales, Bacteroidales, and Anaerolineales, while acetyl-CoA synthetase (EC 6.2.1.1) was mainly produced by Methanobacteriales, Methanosarcinales, and Methanomicrobiales. The critical enzymes acetate CoA-transferase (EC 2.8.3.18) and acetyl-CoA synthetases (EC 6.2.1.13) helped acetyl-CoA change into acetate. Archaea were also the dominant microbes in the 30-, 100-, and 300-year-old PM samples, while cdhA (EC 1.2.7.4), acetyl-CoA synthetase (EC 6.2.1.1), and formate dehydrogenases (EC 1.17.1.9 and EC 1.17.1.10) were the most important enzymes related to methane metabolism. Acetyl-CoA synthetase (EC 6.2.1.1) catalyses the conversion of acetic acid to acetyl-CoA, and formate dehydrogenase (EC 1.17.1.9) catalyses the conversion of formate to $\mathrm{CO}_{2}$, while $\mathrm{EC}$ 1.17.1.10 catalyses the conversion of $\mathrm{CO}_{2}$ to formate.

\section{Metabolic network related to substrate-flavour metabolism in PM microbiota}


To better explain the link among the 20 functional assemblies summarised in Fig. 5, a specific metabolic network in PM microbiota among the nine orders and 30 microbial genera is presented schematically in Fig. 6, showing the pattern of enzyme-coding genes involved in the substrate-flavour metabolic pathway.

\section{Discussion}

CSFL, a fermented and traditional distilled alcoholic beverage, is produced by spontaneous solid-state fermentation containing various microbes and their complex interactions. The standard production of Chinese liquor involves four steps, including cooking, saccharification, alcohol fermentation, and distillation, using grain as the main raw material. In CSFL production, the core microorganisms in PM play important roles in the characteristics of flavour metabolites and determine the quality and safety of CSFL to a large degree.

PM cellar is a microbial ecosystem that provides a natural model for a broader understanding of microbiota composition and succession. Combining 16S rRNA, ITS full-length sequencing, metabonomics, and metagenomic analysis may be valuable for elucidating the microbial community composition, differential metabolites, and the relationship between the microorganisms and the metabolites in PM samples. In our study, sequencing data revealed that PM microbiomes are commonly dominated by bacteria such as Firmicutes, Euryarchaeota, Bacteroidetes, Chloroflexi, Petrimonas, Proteiniphilum, Prevotella, Bellilinea, Caproiciproducens, Clostridium, and Lactobacillus and by archaea such as Methanobacterium and Methanobrevibacter (Fig. 3). Previous studies have shown that prokaryotic communities of 30-, 300-, 40-, and 400-year-old PMs were only found to have prokaryotic abundance differences, without prokaryotic structural differences between them ${ }^{[27]}$, and Clostridium, Caproiciproducens, Syntrophomonas, Sedimentibacter, and Methanobrevibacter were clearly the predominant microorganisms in mature $\mathrm{PM}^{[28,}$ 29]. The difference in the PM bacterial groups in different studies could be explained by a combination of methodological and environmental factors ${ }^{[30]}$. Along with the relatively steady eco-environment and microbiota structure formed through long-term fermentation progress, Caproiciproducens, Prevotella, and Lactobacillus were identified as the most important bacteria at the bottom of the pit, while Clostridium, Bellilinea, and Proteiniphilum showed a higher relative abundance in the Huangshui fluid of the pit. The accumulation of Lactobacillus, Clostridium, and other acid-producing microorganisms at the bottom of the pit leads to an increase in organic acid content (Fig. 2). It is notable that the relative abundance of Methanobacterium and Methanobrevibacterwas $1.44 \%$ to $4.58 \%$ and $2.54 \%$ to $34.42 \%$ in our PM samples, respectively, but the average abundance of Methanobrevibacter was less than $0.05 \%$ in new PM samples in a previous study ${ }^{[31]}$. Low $\mathrm{pH}$ inhibits methanogenic activity in anaerobic biological processes $^{[32]}$. The archaeal genera in PM can utilise $\mathrm{H}_{2}$, acetate, methanol, and methylamine ${ }^{[33]}$. In addition to fermentative bacteria, methanogens can enhance organic acid production through syntrophic interactions $^{[34]}$. 
For fungal microbiota, we did not detect fungi in the 30-, 100-, and 300-year-old PM samples. Previous studies have shown that fungi were not detected in the older PM and were only detected in the new cellars that were used for 2 years $^{[18,35]}$. There are several possible reasons for this: (1) the long fermentation environment is not suitable for the growth of fungi, and oxygen deficiency in the inner compartment partially leads to a low survival rate of fungi, especially Aspergillus ${ }^{[36]}$. (2) The presence of fungi in PM can also be attributed to the addition of the Daqu starter. It is known that the Daqu starter contains abundant microbes such as Lactobacillus, Pseudomonas, Aspergillus, Saccharomyces, Wickerhamomyces, and Pichia, and enzymes such as $\alpha$-amylase, $\beta$-amylase, glucoamylase, and proteases ${ }^{[37,38]}$. During long-term use of the pit, microbes may undergo exchanges between the PM and fermented grains during the fermentation process of the raw material. Therefore, bacteria are more dominant than fungi in older PM samples ${ }^{[9]}$.

\section{Function enzymes related to substrate degradation pathways}

Glycolysis is a central pathway in carbohydrate metabolism and is regarded as a feeder that prepares glucose for further catabolism and energy conservation ${ }^{[39]}$ and has many steps that lead to the catabolism of glucose into pyruvate ${ }^{[40]}$, which diverges according to the availability of oxygen. Thus, the most dominant raw material synthesising the four dominant acids in PM is starch. In our study, the nine orders were all related to starch and sucrose metabolism and glucose utilisation pathway. Moreover, the absolute abundance of Clostridiales, Bacillales, and Lactobacillales were highest in the 300-year-old $\mathrm{PM}$, suggesting that the amount of starch in the 300-year-old PM samples, which turned into the four dominant acids, was much greater than in the 30- and 100-year-old PM samples. The potential capacity for the comprehensive utilisation of different substrates may contribute to their dominance in the entire microbial community.

\section{Function enzymes related to flavour generation pathways}

Like beer and wine, the synergistic effect of microorganisms is thought to be responsible for the production of various flavour compounds, such as CA, butyric acid, acetic acid, lactic acid, and alcohols ${ }^{[3 \text {, }}$ ${ }^{41]}$, in CSFL production ${ }^{[42]}$. Previous studies have shown that pyruvate, ethanol, glutamate, and 4aminobutanoate are related to the four dominant acid syntheses, especially pyruvate and ethanol ${ }^{[14,43]}$. Thus, investigating the ethanol and organic acid metabolism pathways and the core enzymes is vital for improving CSFL quality. Ethanol is the main component of CSFL and is mainly generated through the conversion of pyruvate to acetaldehyde and reduction to ethanol. Some genera such as Bacteroides, Porphyromonas, and Sedimentibacter have been reported to produce succinic acid, propionic acid, and alcohols by using starch and glucose ${ }^{[44]}$. Increasing the content of ethyl CA is an effective method for improving the quality of CSFL. The common synthesis method of CA can occur via carboxylic acid chain elongation using ethanol as an electron donor ${ }^{[45]}$. Acyl-CoA hydrolase is the final enzyme in CA generation and has been previously observed to be enriched within Clostridium spp., indicating that the Clostridium genus was the most important microbiota participating in CA production. Generally, some 
of the most CA-producing Clostridium species include C. kluyveri ${ }^{[6]}$ and Clostridium sp. BS-1 ${ }^{[46]}$. In addition, the $\mathrm{CA}$ production is a process of $\mathrm{H}_{2}$ and $\mathrm{CO}_{2}$ production ${ }^{[47]}$, and $C$. ljungdahlii could synthesize acetic acid and ethanol from $\mathrm{H}_{2}$ and $\mathrm{CO}_{2}$ produced by $C$. clarkil ${ }^{[48]}$. Concurrently, the presence of hydromethanogens and CA-producing bacteria can lead to interspecific hydrogen transfer, which is beneficial to increase CA production ${ }^{[14]}$. Thus, the co-occurrence of the high abundance of Clostridium, Methanobacterium, and Methanobrevibacter may indicate efficient CA production.

Lactic acid not only can inhibit some bacteria but also is an ethyl lactate precursor ${ }^{[49]}$, and it contributes to the formation of other flavour substances ${ }^{[27]}$. Clostridiales (mainly Clostridium) and Lactobacillales (mainly Lactobacillus) were the important potential orders for producing lactic acid in our study. Lactobacillus produces lactic acids from sugar by homofermentative metabolism or produces alcohols in addition to lactic acid via heterofermentative metabolism ${ }^{[50]}$. In addition, Idh (EC 1.1.2.3, EC 1.1.2.4) could catalyse too much lactic acid to pyruvate and then generate acetic acid, butyric acid, and $\mathrm{CA}^{[51]}$, which could contribute to the recovery of $\mathrm{pH}$ of $\mathrm{PM}$ and enhance the production of organic acids. Moreover, the decrease in lactic acid is due to its catalysis not only to pyruvate, but also to acetic acid through EC 1.13.12.4. Excess lactate can lead to high levels of ethyl lactate, which can deteriorate CSFL quality ${ }^{[8]}$. Key genes (e.g., alcohol dehydrogenase and L-lactate dehydrogenase) involved in reverse $\beta$-oxidation with both ethanol and lactate as substrates were found in PM samples, indicating that the PM microbiome possesses metabolic potential for CA production from ethanol and lactate. Our results verified the importance of lactic acid in CA production. Lactobacillus was positively correlated with CA (Additional file: Figure S5), because lactic acid is mainly produced by Lactobacillus and converted into CA by Caproiciproducens ${ }^{[52]}$.

Butyric acid, one of the crucial flavour substances in CSFL, is synthesised through two alternative pathways, namely butyrate kinase (EC 2.7.2.7) and butyryl-CoA:acetate CoA-transferase (EC 2.8.3.8) [53]. A previous study showed that Clostridium was most likely involved in butyrate production through the buk pathway in $\mathrm{PM}^{[14]}$. In our study, besides Clostridiales and Bacteroidales, we also found that Synergistales (mainly Aminobacterium) have the ability to produce enzymes of butyrate kinase (EC 2.7.2.7) and butyryl-CoA: acetate CoA-transferase (EC 2.8.3.8). In addition, butyric acid can provide more precursors for CA synthesis ${ }^{[14]}$. For example, Sedimentibacter and Aminobacterium can ferment amino acids to acetic and butyric acids ${ }^{[54,55]}$. Butanoate metabolism and pyruvate metabolism pathways were related to the production of CA, butyric acid, and lactic acid, and two metabolites, malic acid and succinic acid, were enriched, indicating their importance for the production of organic acids.

Ethyl acetate produced by acetic acid was also important for the formation of flavour in CSFL, and in addition to L-lactic acid, more acetic acid was produced by acetyl-CoA through the catalytic action of pct (EC 2.8.3.1) and acdA (EC 6.2.1.13). The major genus sources of butyrate kinase (EC 2.8.3.1) were Clostridium and Lactobacillus, which had a high absolute abundance in the 300-year-old cell, while the major genus sources of acdA (EC 6.2.1.13) were Methanosarcina, Methanobacterium, Methanobrevibacter, Bellilinea, and Syntrophomonas. Syntrophomonas can degrade long-chain fatty 
acids $\left(\mathrm{C}_{4}-\mathrm{C}_{8}\right)$ into acetic acid, propionic acid, and $\mathrm{H}_{2}{ }^{[56]}$. Furthermore, specific members of the Clostridium species were also identified with acetate biosynthesis from various substrates, such as sugar, starch, and cellulose.

Methane metabolism is also an important pathway during pit fermentation because it can enhance the production of organic acids during fermentation. Methanosarcina co-cultured with bacteria can

metabolise carbon dioxide to methan ${ }^{[57]}$. Methanobrevibacter can produce $\mathrm{CH}_{4}$ from $\mathrm{H}_{2}$ and $\mathrm{CO}_{2}{ }^{[58]}$, and the interspecies hydrogen transfer between archaea and Clostridiaceae (mainly Clostridium) plays an important role in improving the quality of $\mathrm{CSFL}^{[59]}$. In our study, the Methanomicrobiales (mainly Methanoculleus) and Methanobacteriales (mainly Methanobrevibacter and Methanobacterium) were the most abundant microbiota orders participating in methane metabolism. Further, the genera Caloramator and Clostridium, belonging to Clostridiaceae, mainly produced the enzymes EC 5.4.2.12 and 1.17.1.9. In our study, Methanobrevibacter was positively correlated with lactic acid by metabonomics (Additional file: Figure S5), indicating that Methanobrevibacter played an important role in the production of lactic acid.

\section{Environmental factors drive microbiota succession in PM}

Microbial community succession is a complex long-term process. Physicochemical factors are believed to be the main determinants that influence the shift of the microbiota community and structure in the soil and $\mathrm{PM}^{[19]}$. This study revealed that organic acids such as acetic acid, $\mathrm{CA}$, butyric acid, and lactic acid were the most important indicators influencing microbial community structure in the 30-, 100-, and 300year-old PM samples (Fig. 4B). The organic acid content was highest at position 1 of the cellar, especially in the 300-year-old PM samples (Fig. 2). Previous studies revealed that lactic acid and pH were the two important factors influencing microbiota composition in 1-, 10-, 25-, and 50-year-old PM samples. Hartman et al. ${ }^{[60]}$ also found that $\mathrm{pH}$ was the most essential environmental factor influencing the bacterial community structure in soil, and $\mathrm{pH}$ broadly altered the prokaryotic diversity and affected specific taxa, including Acidobacteria and Actinobacteria. In our study, $\mathrm{pH}$ and moisture were stable in the cellars and influenced a few bacteria. The moisture content can affect the microbiota composition, which may be related to the lack of free water available to PM microorganisms. In our study, the moisture content gradually increased with cellar use and was higher in positions 1 and 2 than in other spatial locations, especially for the 100- and 300-year-old cellars. This may be because PM was soaked in Huangshui for a long time during long-term CSFL fermentation, thus leading to a high moisture content. Simultaneously, ammonium nitrogen can provide a source of nitrogen for microbial growth and has an important influence on the abundance of PM microbiota ${ }^{[19]}$. The concentrations of TN and AP increased with the cellar age and showed certain spatial rules; the content of AP and TN in position 1 was higher than in other spatial locations. Therefore, the microbial community structure can be adjusted by adjusting environmental factors such as moisture and $\mathrm{pH}$ of the $\mathrm{PM}$ to achieve the purpose of increasing $\mathrm{CA}$ and decreasing lactic acid content ${ }^{[61]}$. Consequently, a challenge in Chinese liquor production is to achieve a reduction in ethyl lactate synthesis during CSFL production. 
In our study, we investigated the core microbiota composition in PM using sequencing technologies and identified metabolites using GC-MS and LC-MS. Amplicon analysis revealed fundamental information about microbial succession, and RDA revealed positive correlations between the core microbiota and major endogenous factors. The metagenomic data illustrated the effectiveness of the analysis in increasing our understanding of the possible mechanisms responsible for the microbial community structure in PM, providing a reference for further utilisation of PM microbiota genetics and paving the way for the optimisation of CSFL production. However, a limitation of this study is that some microorganisms present throughout the process may have died and left their DNA in the samples. This process requires further exploration via isolation of microbes and functional genomic analysis.

\section{Conclusions}

These cellars have been used for several years without interruption, providing an ideal opportunity to uncover the effect of cellar age on the microbial succession and functional diversity of the microorganisms in PM. Although the different cellars harboured the taxonomically and functionally diverse and abundant microbial community, the functional microbiota related to flavour generation as a hotspot for microbial diversity, genes for flavour generation, and substrate degradation were identified throughout the 30-, 100-, and 300-year-old cellars. However, metagenomic datasets revealed that the pathways and taxa involved in flavour generation and substrate degradation were complex and diverse. Clostridiales (mainly Clostridium) and Bacteroidales (mainly Petrimonas and Proteiniphilum) are major microbiota orders for flavour generation and substrate degradation pathways, while archaea also play an important role, in cooperation with bacteria, in various flavour generation pathways. Our data help elucidate the core microbiota composition, different metabolic roles of microorganisms, and disclose the formation mechanism of PM partial functions, providing a basic theory to support the regulation of Baijiu production.

\section{Methods}

\section{Sample collection}

PM samples were obtained from an SFB-producing enterprise in Luzhou, the south-eastern part of Sichuan Province, China. The cellar was approximately $2.8 \mathrm{~m}$ length, $1.8 \mathrm{~m}$ width, and $2.0 \mathrm{~m}$ height (Fig. 1). We sampled the PM from different fermentation pit cellars that have been used continuously for 30 , 100 , and $300+$ years. We shovelled the PM samples from the bottom of the cellar, under and up the middle site ( $80-100 \mathrm{~cm}$ below the upper edge), and the top site $(0-20 \mathrm{~cm}$ below the upper edge), namely positions $1-4$, respectively. The PM samples were collected at approximately $400 \mathrm{~g}$. After collection, the samples were sufficiently mixed and placed in a sterilising bag, placed in a foam box filled with dry ice, and finally returned to the laboratory for total DNA extraction.

\section{Detection of PM physicochemical properties and organic acids}


The moisture content of PM was measured using a dry/wet weight measurement method after drying at $105^{\circ} \mathrm{C}$ for $6 \mathrm{~h}$. The $\mathrm{pH}$ of fresh PM was determined in a 1:10 (w/v) ratio in deionised water using a pH meter (PB10; Sartorius, Gottingen, Germany). The concentration of available phosphorus (AP) was detected by extracting with ammonium fluoride and hydrochloric acid ${ }^{[62]}$. Total nitrogen was extracted with Seignette salt and ammonium chloride and its concentration measured using UV-visible spectrophotometry. Organic acids such as Caproic acid, butyric acid, and acetic acid in the PM were detected by GC-MS: $1 \mathrm{~g}$ PM samples and $2 \mathrm{~mL} 60 \%$ ethanol were placed in a 5 -mL centrifuge tube with a vortex mixer. After 30 minutes of extraction, the mixture was centrifuged at $10000 \mathrm{rpm}$ for 10 minutes. Afterwards, the supernatant was collected through the membrane for GC-MS analysis. The conditions were as follows: the inlet temperature was $230^{\circ} \mathrm{C}$, the carrier gas was high-purity helium, and the flow rate was $1.0 \mathrm{~mL} / \mathrm{min}$. Moreover, the following gradient conditions were used: a temperature of $35^{\circ} \mathrm{C}$ maintained for $10 \mathrm{~min}$ and then increased to $60^{\circ} \mathrm{C}$ at $4{ }^{\circ} \mathrm{C} / \mathrm{min}$ and maintained for $4 \mathrm{~min}$. The temperature was further increased to $195^{\circ} \mathrm{C}$ at $6^{\circ} \mathrm{C} / \mathrm{min}$ for $20 \mathrm{~min}$. Lactic acid in the PM was quantified using HPLC: $1 \mathrm{~g}$ PM samples and $2 \mathrm{~mL}$ ultra-pure water were placed in a 5-mL centrifuge tube, and after 30 min of extraction, the supernatant was collected through the membrane for HPLC analysis, as described previously ${ }^{[63]}$.

\section{DNA extraction, 16S rRNA gene, and ITS amplicon sequencing}

Genomic DNA of PM samples was extracted using a FastDNA spin kit for soil, according to the manufacturer's instructions. The concentration and purity of DNA were determined using a NanoDrop 2000 UV-vis spectrophotometer (Thermo Scientific, Wilmington, USA) and 1\% agarose gel electrophoresis, and the library was constructed and sequenced after passing the test. To analyse the microbial community, a target variable region of the small ribosomal subunit RNA gene was PCR-amplified with the KAPA HiFi Ready Mix (Kapa Biosystems, KK2602). The universal primers (16S: Forward Primers sequences AGRGTTYGATYMTGGCTCAG, Reverse primers sequences RGYTACCTTGTTACGACTT and ITS: Forward Primers sequences TCCGTAGGTGAACCTGCGG, Reverse primers sequences TCCTCCGCTTATTGATATGC) with the barcodes were used to amplify the bacterial 16S rRNA and ITS regions of fungal rRNA, respectively. The PCR product was then purified by $0.6 x$ AMPure PB Beads, and the library was constructed using the SMRTbell Express Template Prep Kit 2.0 (PacBio) with damage repair, end repair, A-tailing, and ligation of sequencing adapters. The sequencing was performed on a PacBio Sequel II instrument with Sequencing Primer V4 and Sequel II Binding Kit 2.1 in Grandomics at Wuhan Biotechnology Co., Ltd (Wuhan, China).

\section{S rDNA and ITS amplicon data analyses}

The original FASTQ file was processed using QIIME software ${ }^{[64]}$, and the low-quality sequences were filtered out. After analysing the sequencing data, the SILVA database (v13.2) was used to compare the 16S rRNA and ITS gene sequences to determine the taxonomic status of the corresponding microbes. QIIME software was used to define sequence similarity $>97 \%$ as an operational taxonomic unit (OTU) ${ }^{[65]}$. Only OTUs containing at least five reads were considered valid in this study, and we selected a 
representative sequence of each OTU for taxonomic analysis. The alpha diversity index was calculated to analyse species richness and uniformity in the samples, after which the Shannon and Chao1 indices of the samples were determined to obtain the diversity of the community and the number of OTUs in the samples. The genus-level clustering results for each sample were used as inputs for LEfSe analysis. Finally, the beta diversity index was determined to analyse the heterogeneity of community composition between samples.

\section{Metagenomic sample preparation, processing, and assembly of metagenomic sequencing data}

Metagenomic shotgun sequencing libraries were constructed and sequenced by Beijing Novogene Bioinformatics Technology Co. Ltd. Briefly, $8 \mu \mathrm{g}$ DNA per sample was used as the input material for DNA sample preparations. Sequencing libraries were generated using the Ligation Sequencing Kit (SQKLSK109) following the manufacturer's recommendations. DNA was disrupted with a Megaruptor (Diagenode, NJ, USA), and fragments greater than $10 \mathrm{~kb}$ were screened using BluePippin. After end repair and addition of $A$ tail, the barcodes were added, and then the fragment length was detected. Samples with different barcodes were mixed in equimolar amounts, and the linker and template were purified. DNA concentration was measured using Qubit to complete the preparation of the DNA library. The barcoded samples were clustered using the PromethION Flow Cell Priming Kit (EXPFLP001.PRO.6) according to the manufacturer's instructions. After sequencing, the clean data were assembled and analysed by Flye (version: 2.4.2 ${ }^{[66]}$ after data pre-processing, and the assembled scaftigs were checked with the clean data of every sample. Additionally, all clean data samples were compared to each scaffold using Bowtie 2.2.4 software to acquire the PE reads not used. Filter fragments shorter than $500 \mathrm{bp}$ in all scaftigs for statistical analysis were generated from a single assembly. Every unigene sequence of PM samples was aligned to bacterial, archaeal, fungal, and viral sequences of the NCBI-NT library using BLASTN ( $E$ value<0.001).

\section{Metabolomic analysis of PM}

The PM samples from positions 1 and 2 were mixed equally to form a new sample termed 'positions under the Huangshui fluid'. The PM samples from positions 3 and 4 were mixed equally to form a new sample termed 'positions above the Huangshui fluid'. Untargeted metabolomic analysis was used to identify the differential metabolites between the different PM samples using GC-MS and LC-MS. GC-MS analysis was performed using a 7890A gas chromatograph (Agilent) coupled to a PEGASUS HT mass selective detector (LECO). LC-MS analysis was performed on an Acquity UPLC system (Thermo Fisher Scientific) coupled with a Q Exactive HFX (Thermo Fisher Scientific). Detailed sample preparation and MS analysis methods are described in Supplementary text $\mathrm{S} 1$.

\section{Statistical analysis}

Origin 2017, 64 Bit, and SPSS 21 software were used for data processing and analysis. A redundancy analysis (RDA) was performed using CANOCO 4.5 software (Microcomputer Power, Ithaca, NY). The statistical significance of the difference between the mean of samples was tested by a one-way analysis 
of variance with the Tukey post hoc test. The cluster analysis and correlation coefficient between microbes and chemical properties were visualised using $R$ software ( $V 3.6 .3$ and $V$ 4.0.5).

\section{Abbreviations}

PM

Pit Mud

LC-MS

liquid chromatograph-mass spectrometer

GC-MS

Gas-chromatography-mass spectrometry

RDA

redundancy analysis

NMDS

Non-metric multidimensional scaling

LEfSe

Linear discriminant analysis Effect Size. $\beta$ NTI:beta nearest taxonomic index

\section{Declarations}

\section{Acknowledgements}

The authors would like to acknowledge and thank Qianqian Yin, Chen Yang, and Yilun Li for their help with PM sampling.

\section{Authors' contributions}

Conceptualization, methodology, formal analysis, investigation, visualization, writing original draft, D.L.R.; Writing - review \& editing, S.P.L.; Data curation, writing -review \& editing, X. H.; Funding acquisition, J.M. The authors read and approved the final manuscript.

\section{Funding}

This work was financially supported by the National Natural Science Foundation of China (32072205, 22138004), Sichuan Science and Technology Program (2021YFS0337), The first phase of the connotation construction of the 14th Five-Year Plan of Tibetan medicine (2021ZYYGH008).

\section{Availability of data and materials}

No obtained data required submission to a public repository. Raw data or further details related to the conducted experiments can be obtained upon request from the corresponding author. 
Not applicable.

\section{Consent for publication}

Not applicable.

\section{Competing interests}

The authors declare that they have no competing interests.

\section{References}

1. Wang XS, Du H, Xu Y. Source tracking of prokaryotic communities in fermented grain of Chinese strong-flavor liquor. Int J Food Microbiol. 2017;244:27-35. https://10.1016/j.ijfoodmicro.2016.12.018.

2. Liu HL, Sun BG. Effect of Fermentation Processing on the Flavor of Baijiu. J Agric Food Chem. 2018;66(22):5425-32. https://10.1021/acs.jafc.8b00692.

3. Chen L, Li YZ, Jin L, He L, Ao XL, Liu SL, et al. Analyzing bacterial community in pit mud of Yibin Baijiu in China using high throughput sequencing. Peerj. 2020;8(1):e9122. https://10.7717/peerj.9122.

4. Zou W, Zhao C, Luo H. Diversity and Function of Microbial Community in Chinese Strong-Flavor Baijiu Ecosystem: A Review. Front Microbiol. 2018;9:671. https://10.3389/fmicb.2018.00671.

5. Wang CD, Chen Q, Wang Q, Li CH, Leng YY, Li SG, et al. Long-term batch brewing accumulates adaptive microbes, which comprehensively produce more flavorful Chinese liquors. Food Res Int. 2014;62:894-901. https://10.1016/j.foodres.2014.05.017.

6. Hu XL, Du H, Xu Y. Identification and quantification of the caproic acid-producing bacterium Clostridium kluyveri in the fermentation of pit mud used for Chinese strong-aroma type liquor production. Int J Food Microbiol. 2015;214:116-22. https://10.1016/j.ijfoodmicro.2015.07.032.

7. Ding XF, Wu CD, Huang J, Li H, Zhou RQ. Eubacterial and archaeal community characteristics in the man-made pit mud revealed by combined PCR-DGGE and FISH analyses. Food Res Int. 2014;62:1047-53. https://10.1016/j.foodres.2014.05.045.

8. He GQ, Huang J, Wu CD, Jin Y, Zhou RQ. Bioturbation effect of fortified Daqu on microbial community and flavor metabolite in Chinese strong-flavor liquor brewing microecosystem. Food Res Int. 2020;129:108851. https://10.1016/j.foodres.2019.108851.

9. Zheng J, Liang R, Zhang L, Wu C, Zhou R, Liao X. Characterization of microbial communities in strong aromatic liquor fermentation pit muds of different ages assessed by combined DGGE and PLFA analyses. Food Res Int. 2013;54(1):660-6. https://10.1016/j.foodres.2013.07.058.

10. Tracy BP, Jones SW, Fast AG, Indurthi DC, Papoutsakis ET. Clostridia: the importance of their exceptional substrate and metabolite diversity for biofuel and biorefinery applications. Curr Opin Biotechnol. 2012;23(3):364-81. https://10.1016/j.copbio.2011.10.008. 
11. Wang HY, Xu Y. Effect of temperature on microbial composition of starter culture for Chinese light aroma style liquor fermentation. Lett Appl Microbiol. 2015;60(1):85-91. https://10.1111/lam.12344.

12. Zou W, Ye G, Zhang K. Diversity. Function, and Application of Clostridium in Chinese Strong Flavor Baijiu Ecosystem: A Review. J Food Sci. 2018;83(5):1193-9. https://10.1111/1750-3841.14134.

13. Cavalcante WdA, Leitão RC, Gehring TA, Angenent LT, Santaella ST. Anaerobic fermentation for ncaproic acid production: A review. Process Biochem. 2017;54:106-19. https://10.1016/j.procbio.2016.12.024.

14. Tao Y, Wang X, Li X, Wei N, Jin H, Xu Z, et al. The functional potential and active populations of the pit mud microbiome for the production of Chinese strong-flavour liquor. Microb Biotechnol. 2017;10(6):1603-15. https://10.1111/1751-7915.12729.

15. Gao Z, Wu Z, Zhang W. Effect of Pit Mud on Bacterial Community and Aroma Components in Yellow Water and Their Changes during the Fermentation of Chinese Strong-Flavor Liquor. Foods. 2020;9(3). https://10.3390/foods9030372.

16. Xiong ZS. Origin and Development of Chinese First Fermentation Pit--Record of Luzhou Laojiao Liquor. Liquor-making Science \& Technology; 2001.

17. Xu Y, Wang D, Fan WL, Mu XQ, Chen J. Traditional Chinese biotechnology. Adv Biochem Eng Biotechnol. 2010;122:189-233. https://10.1007/10_2008_36.

18. Zhao JS, Zheng J, Zhou RQ, Shi B. Microbial community structure of pit mud in a Chinese strong aromatic liquor fermentation pit. J Inst Brew. 2012;118(4):356-60. https://10.1002/jib.52.

19. Hu X, Du H, Ren C, Xu Y. Illuminating Anaerobic Microbial Community and Cooccurrence Patterns across a Quality Gradient in Chinese Liquor Fermentation Pit Muds. Appl Environ Microbiol. 2016;82(8):2506-15. https://10.1128/AEM.03409-15.

20. Yin Q, Tao Y, Zhu X, Zhou Y, He X, Cheng L, et al. Clostridium liquoris sp. nov., isolated from a fermentation pit used for the production of Chinese strong-flavoured liquor. Int J Syst Evol Microbiol. 2016;66(2):749-54. https://10.1099/ijsem.0.000787.

21. Guo MY, Huo DQ, Ghai R, Rodriguez-Valera F, Shen $\mathrm{CH}$, Zhang N, et al. Metagenomics of ancient fermentation pits used for the production of chinese strong-aroma liquor. Genome Announc. 2014;2(5). https://10.1128/genomeA.01045-14.

22. Liang $\mathrm{H}$, Luo Q, Zhang A, Wu Z, Zhang W. Comparison of bacterial community in matured and degenerated pit mud from ChineseLuzhou-flavourliquor distillery in different regions. J Inst Brew. 2016;122(1):48-54. https://10.1002/jib.296.

23. Almeida A, Mitchell AL, Boland M, Forster SC, Gloor GB, Tarkowska A, et al. A new genomic blueprint of the human gut microbiota. Nature. 2019;568(7753):499-504. https://10.1038/s41586-019-09651.

24. Stewart RD, Auffret MD, Warr A, Wiser AH, Press MO, Langford KW, et al. Assembly of 913 microbial genomes from metagenomic sequencing of the cow rumen. Nat Commun. 2018;9(1):870. https://10.1038/s41467-018-03317-6. 
25. Graham EB, Knelman JE, Schindlbacher A, Siciliano S, Breulmann M, Yannarell A, et al. Microbes as Engines of Ecosystem Function: When Does Community Structure Enhance Predictions of Ecosystem Processes? Front Microbiol. 2016;7:214. https://10.3389/fmicb.2016.00214.

26. Chai LJ, Xu PX, Qian W, Zhang XJ, Ma J, Lu ZM, et al. Profiling the Clostridia with butyrate-producing potential in the mud of Chinese liquor fermentation cellar. Int J Food Microbiol. 2019;297:41-50. https://10.1016/j.ijfoodmicro.2019.02.023.

27. Huimin Z, Yajing M, Yanli W, Qingwu Z, Anjun L, Guoying L, et al. Prokaryotic communities in multidimensional bottom-pit-mud from old and young pits used for the production of Chinese Strong-Flavor Baijiu. Food Chem. 2020;312:126084-4. https://10.1016/j.foodchem.2019.126084.

28. Luo Q, Liu C, Wu Z, Wang H, Li W, Zhang K, et al. Monitoring of the prokaryotic diversity in pit mud from aLuzhou-flavourliquor distillery and evaluation of two predominant archaea using qPCR assays. J Inst Brew. 2014;120(3):253-61. https://10.1002/jib.132.

29. Liang H, Li W, Luo Q, Liu C, Wu Z, Zhang W. Analysis of the bacterial community in aged and aging pit mud of Chinese Luzhou-flavour liquor by combined PCR-DGGE and quantitative PCR assay. J Sci Food Agric. 2015;95(13):2729-35. https://10.1002/jsfa.7013.

30. Liu MK, Tang YM, Guo XJ, Zhao K, Tian XH, Liu Y, et al. Deep sequencing reveals high bacterial diversity and phylogenetic novelty in pit mud from Luzhou Laojiao cellars for Chinese strong-flavor Baijiu. Food Res Int. 2017;102:68-76. https://10.1016/j.foodres.2017.09.075.

31. Liu MK, Tang YM, Guo XJ, Zhao K, Penttinen P, Tian XH, et al. Structural and Functional Changes in Prokaryotic Communities in Artificial Pit Mud during Chinese Baijiu Production. Msystems. 2020;5(2):e00829-19. https://10.1128/mSystems.00829-19.

32. Kessel J, Russell JB. The effect of pH on ruminal methanogenesis. Blackwell Publishing Ltd. 1996;20(4):205-10. https://10.1016/0168-6496(96)00030-x.

33. Demirel B, Scherer P. The roles of acetotrophic and hydrogenotrophic methanogens during anaerobic conversion of biomass to methane: a review. Rev Environ Sci Biotechnol. 2008;7(2):173-90. https://10.1007/s11157-008-9131-1.

34. Felchner-Zwirello M, Winter J, Gallert C. Interspecies distances between propionic acid degraders and methanogens in syntrophic consortia for optimal hydrogen transfer. Appl Microbiol Biotechnol. 2013;97(20):9193-205. https://10.1007/s00253-012-4616-9.

35. Zhang M, Wu X, Mu D, Yang W, Jiang S, Sun W, et al. Profiling the effects of physicochemical indexes on the microbial diversity and its aroma substances in pit mud. Lett Appl Microbiol. 2020;71(6):66778. https://10.1111/lam.13380.

36. Hall LA, Denning DW. Oxygen Requirements of Aspergillus Species. J Med Microbiol. 1994;41(5):311-5. https://Doi 10.1099/00222615-41-5-311.

37. Wang HY, Gao YB, Fan QW, Xu Y. Characterization and comparison of microbial community of different typical Chinese liquor Daqus by PCR-DGGE. Lett Appl Microbiol. 2011;53(2):134-40. https://10.1111/j.1472-765X.2011.03076.x. 
38. Zheng XW, Tabrizi MR, Nout M, Han BZJ. Daqu-. A Traditional Chinese Liquor Fermentation Starter. J Inst Brew. 2011;117(1):82-90. https://https://doi.org/10.1002/j.2050-0416.2011.tb00447.x.

39. Lenzen S. A fresh view of glycolysis and glucokinase regulation: history and current status. J Biol Chem. 2014;289(18):12189-94. https://10.1074/jbc.R114.557314.

40. Serina J, Fernandes MX, Castilho PC. Effects of hydroxycinnamic acids on the glycolysis pathway. S. Afr J Bot. 2019;120:219-29. https://10.1016/j.sajb.2018.06.016.

41. Lu MM, Zhou WC, Ji F, Wu JF, Nie Y, Ren C, et al. Profiling prokaryotic community in pit mud of Chinese strong-aroma type liquor by using oligotrophic culturing. Int J Food Microbiol. 2021;337. https://10.1016/j.jijoodmicro.2020.108951.

42. Bokulich NA, Bamforth CW, Mills DA. A Review of Molecular Methods for Microbial Community Profiling of Beer and Wine. J Am Soc Brew Chem. 2018;70(3):150-62. https://10.1094/asbcj-20120709-01.

43. Zhou W, Liao ZM, Wu ZY, Suyama T, Zhang WX. Analysis of the difference between aged and degenerated pit mud microbiome in fermentation cellars for Chinese Luzhou-flavor baijiu by metatranscriptomics. J Sci Food Agric. 2021;101(11):4621-31. https://10.1002/jsfa.11105.

44. Kawamura Y, Kuwabara S, Kania SA, Kato H, Hamagishi M, Fujiwara N, et al. Porphyromonas pogonae sp. nov., an anaerobic but low concentration oxygen adapted coccobacillus isolated from lizards (Pogona vitticeps) or human clinical specimens, and emended description of the genus Porphyromonas Shah and Collins 1988. Syst Appl Microbiol. 2015;38(2):104-9. https://10.1016/j.syapm.2014.11.004.

45. He G, Huang J, Wu C, Jin Y, Zhou R. Bioturbation effect of fortified Daqu on microbial community and flavor metabolite in Chinese strong-flavor liquor brewing microecosystem. Food Res Int. 2020;129:108851. https://10.1016/j.foodres.2019.108851.

46. Jeon BS, Kim BC, Um Y, Sang BI. Production of hexanoic acid from D-galactitol by a newly isolated Clostridium sp. BS-1. Appl Microbiol Biotechnol. 2010;88(5):1161-7. https://10.1007/s00253-0102827-5.

47. Ding HB, Tan GY, Wang JY. Caproate formation in mixed-culture fermentative hydrogen production. Bioresour Technol. 2010;101(24):9550-9. https://10.1016/j.biortech.2010.07.056.

48. Richter H, Molitor B, Diender M, Sousa DZ, Angenent LT. A Narrow pH Range Supports Butanol, Hexanol, and Octanol Production from Syngas in a Continuous Co-culture of Clostridium ljungdahlii and Clostridium kluyveri with In-Line Product Extraction. Front Microbiol 2016;7. https://10.3389/fmicb.2016.01773.

49. Jin G, Zhu Y, Xu Y. Y. Mystery behind Chinese liquor fermentation. Trends Food Sci Technol. 2017;63:18-28. https://https://doi.org/10.1016/j.tifs.2017.02.016.

50. Papagianni M. Metabolic engineering of lactic acid bacteria for the production of industrially important compounds. Comput Struct Biotechnol J. 2012;3:e201210003. https://10.5936/csbj.201210003. 
51. Tao Y, Zhu XY, Wang H, Wang Y, Li XZ, Jin H, et al. Complete genome sequence of Ruminococcaceae bacterium CPB6: A newly isolated culture for efficient n-caproic acid production from lactate. $\mathrm{J}$ Biotechnol. 2017;259:91-4. https://10.1016/j.jbiotec.2017.07.036.

52. Zhu X, Zhou Y, Wang Y, Wu T, Li X, Li D, et al. Production of high-concentration n-caproic acid from lactate through fermentation using a newly isolated Ruminococcaceae bacterium CPB6. Biotechnol Biofuels. 2017;10:102. https://10.1186/s13068-017-0788-y.

53. Chai L-J, Lu Z-M, Zhang X-J, Ma J, Xu P-X, Qian W, et al. Zooming in on Butyrate-Producing Clostridial Consortia in the Fermented Grains of Baijiu via Gene Sequence-Guided Microbial Isolation. Front Microbiol. 2019;10. https://10.3389/fmicb.2019.01397.

54. Hamdi O, Ben Hania W, Postec A, Bouallagui H, Hamdi M, Bonin P, et al. Aminobacterium thunnarium sp. nov., a mesophilic, amino acid-degrading bacterium isolated from an anaerobic sludge digester, pertaining to the phylum Synergistetes. Int J Syst Evol Microbiol. 2015;65(Pt 2):609-14. https://10.1099/ijs.0.068965-0.

55. Imachi H, Sakai S, Kubota T, Miyazaki M, Saito Y, Takai K. Sedimentibacter acidaminivorans sp. nov., an anaerobic, amino-acid-utilizing bacterium isolated from marine subsurface sediment. Int J Syst Evol Microbiol. 2016;66(3):1293-300. https://10.1099/ijsem.0.000878.

56. Mclnerney. MJ, Bryant MP., Hespell. RB. J W, Costerton.. Syntrophomonas wolfei gen. nov. sp. nov., an Anaerobic, Syntrophic, Fatty Acid-Oxidizing Bacterium. Appl Environ Microbiol. 1981;41(4). https://10.1128/aem.41.4.1029-1039.1981.

57. Rotaru AE, Shrestha PM, Liu F, Markovaite B, Chen S, Nevin KP, et al. Direct interspecies electron transfer between Geobacter metallireducens and Methanosarcina barkeri. Appl Environ Microbiol. 2014;80(15):4599-605. https://10.1128/AEM.00895-14.

58. Lee JH, Kumar S, Lee GH, Chang DH, Rhee MS, Yoon MH, et al. Methanobrevibacter boviskoreani sp. nov., isolated from the rumen of Korean native cattle. Int J Syst Evol Microbiol. 2013;63(Pt 11):4196201. https://10.1099/ijs.0.054056-0.

59. Ding X, Wu C, Huang J, Zhou R. Interphase microbial community characteristics in the fermentation cellar of Chinese Luzhou-flavor liquor determined by PLFA and DGGE profiles. Food Res Int. 2015;72:16-24. https://10.1016/j.foodres.2015.03.018.

60. Hartman WH, Richardson CJ, Vilgalys R, Bruland, GLJPotNAoSotUSoA. Environmental and anthropogenic controls over bacterial communities in wetland soils. P NATL ACAD SCI USA. 2008;105(46):17842-7. https://10.1073/pnas.0808254105.

61. Jiang J, Liu Y, Li H, Yang Q, Wu Q, Chen S, et al. Modeling and Regulation of Higher Alcohol Production through the Combined Effects of the C/N Ratio and Microbial Interaction. J Agric Food Chem. 2019;67(38):10694-701. https://10.1021/acs.jafc.9b04545.

62. Liu X, Meng W, Liang G, Li K, Xu W, Huang L, et al. Available phosphorus in forest soil increases with soil nitrogen but not total phosphorus: evidence from subtropical forests and a pot experiment. PLoS One. 2014;9(2):e88070. https://10.1371/journal.pone.0088070. 
63. Gong M, Zhou ZL, Yu YJ, Liu SP, Zhu SH, Jian DZ, et al. Investigation of the 5-hydroxymethylfurfural and furfural content of Chinese traditional fermented vinegars from different regions and its correlation with the saccharide and amino acid content. Lwt. 2020;124. https://10.1016/j.Iwt.2020.109175.

64. Caporaso JG, Kuczynski J, Stombaugh J, Bittinger K, Bushman FD, Costello EK, et al. QIIME allows analysis of high-throughput community sequencing data. Nat Methods. 2010;7(5):335-6. https://10.1038/nmeth.f.303.

65. Edgar RC. Search and clustering orders of magnitude faster than BLAST. Bioinformatics. 2010;26(19):2460-1. https://10.1093/bioinformatics/btq461.

66. Kolmogorov M, Yuan J, Lin Y, Pevzner PA. Assembly of long, error-prone reads using repeat graphs. Nat Biotechnol. 2019;37(5):540-6. https://10.1038/s41587-019-0072-8.

\section{Tables}


Table 1

The spatiotemporal physicochemical properties difference of PM during CSFL fermentation

\begin{tabular}{|lllll|}
\hline & Position-1 & Position-2 & Position-3 & Position-4 \\
\hline Moisture-30-year & $38.21 \pm 4.7 \mathrm{a} / \mathrm{A}$ & $39.1 \pm 5.95 \mathrm{a} / \mathrm{A}$ & $37.95 \pm 2.97 \mathrm{a} / \mathrm{A}$ & $38.27 \pm 4.06 \mathrm{a} / \mathrm{A}$ \\
\hline 100-year & $43.48 \pm 2.47 \mathrm{a} / \mathrm{A}$ & $41.97 \pm 1.48 \mathrm{a} / \mathrm{A}$ & $39.91 \pm 2.45 \mathrm{ab} / \mathrm{A}$ & $38.11 \pm 0.28 \mathrm{~b} / \mathrm{A}$ \\
\hline 300-year & $45.79 \pm 1.31 \mathrm{a} / \mathrm{A}$ & $45.08 \pm 1.13 \mathrm{a} / \mathrm{A}$ & $43.12 \pm 3.32 \mathrm{a} / \mathrm{A}$ & $36.05 \pm 5.06 \mathrm{~b} / \mathrm{A}$ \\
\hline pH-30-year & $6.69 \pm 0.19 \mathrm{a} / \mathrm{A}$ & $6.02 \pm 0.57 \mathrm{a} / \mathrm{A}$ & $6.72 \pm 0.22 \mathrm{a} / \mathrm{A}$ & $4.53 \pm 0.53 \mathrm{~b} / \mathrm{A}$ \\
\hline 100-year & $6.38 \pm 0.38 \mathrm{a} / \mathrm{A}$ & $6.37 \pm 0.31 \mathrm{a} / \mathrm{A}$ & $5.66 \pm 0.83 \mathrm{a} / \mathrm{A}$ & $6.26 \pm 0.07 \mathrm{a} / \mathrm{B}$ \\
\hline 300-year & $6.33 \pm 0.12 \mathrm{a} / \mathrm{A}$ & $6.5 \pm 0.4 \mathrm{a} / \mathrm{A}$ & $6.52 \pm 0.51 \mathrm{a} / \mathrm{A}$ & $6.63 \pm 0.41 \mathrm{a} / \mathrm{B}$ \\
\hline TN (g/Kg)-30-year & $3.92 \pm 0.34 \mathrm{a} / \mathrm{B}$ & $3.45 \pm 1.15 \mathrm{a} / \mathrm{A}$ & $4.15 \pm 0.19 \mathrm{a} / \mathrm{A}$ & $2.67 \pm 0.66 \mathrm{~b} / \mathrm{A}$ \\
\hline 100-year & $3.74 \pm 0.15 \mathrm{~b} / \mathrm{B}$ & $2.7 \pm 0.39 \mathrm{a} / \mathrm{A}$ & $3.48 \pm 0.24 \mathrm{~b} / \mathrm{A}$ & $1.94 \pm 0.03 \mathrm{c} / \mathrm{A}$ \\
\hline 300-year & $2.82 \pm 0.49 \mathrm{a} / \mathrm{A}$ & $2.9 \pm 0.38 \mathrm{a} / \mathrm{A}$ & $2.97 \pm 1.43 \mathrm{a} / \mathrm{A}$ & $2.22 \pm 0.62 \mathrm{~b} / \mathrm{A}$ \\
\hline AP (g/Kg)-30-year & $4.57 \pm 1.74 \mathrm{a} / \mathrm{A}$ & $3.25 \pm 0.33 \mathrm{a} / \mathrm{A}$ & $4.19 \pm 2.36 \mathrm{a} / \mathrm{A}$ & $1.87 \pm 0.01 \mathrm{a} / \mathrm{A}$ \\
\hline 100-year & $5.38 \pm 1.36 \mathrm{a} / \mathrm{A}$ & $5.69 \pm 0.27 \mathrm{a} / \mathrm{B}$ & $3.47 \pm 0.92 \mathrm{~b} / \mathrm{A}$ & $1.94 \pm 0.03 \mathrm{~b} / \mathrm{B}$ \\
\hline 300-year & $4.07 \pm 1.32 \mathrm{~b} / \mathrm{A}$ & $3.34 \pm 0.77 \mathrm{ab} / \mathrm{A}$ & $1.93 \pm 0.59 \mathrm{a} / \mathrm{A}$ & $1.96 \pm 0.23 \mathrm{a} / \mathrm{A}$ \\
\hline Simpson & $0.9 \pm 0.05 \mathrm{a}$ & $0.95 \pm 0.02 \mathrm{a}$ & $0.9 \pm 0.07 \mathrm{a}$ & $0.92 \pm 0.05 \mathrm{a}$ \\
\hline Shannon & $5.41 \pm 0.96 \mathrm{a}$ & $5.96 \pm 0.71 \mathrm{a}$ & $5.12 \pm 0.93 \mathrm{a}$ & $5.48 \pm 0.86 \mathrm{a}$ \\
\hline Coverage (\%) & $92.35 \pm 3.88$ & $93.65 \pm 1.86$ & $94.05 \pm 1.84$ & $91.72 \pm 4.96$ \\
\hline $\begin{array}{l}\text { All data are presented as mean } \pm \text { standard deviations ( } \mathrm{n}=3 \text { ), Lowercase letters (a,b,c,d) represent a } \\
\text { horizontal comparison of different spatial positions, upper case letters (A,B,C,D) represent a vertical } \\
\text { comparison of pits in different years. }\end{array}$ & & & \\
\hline AP: Available phosphorus & & & \\
\hline TN: Total Nigroten & & & & \\
\hline
\end{tabular}

\section{Figures}




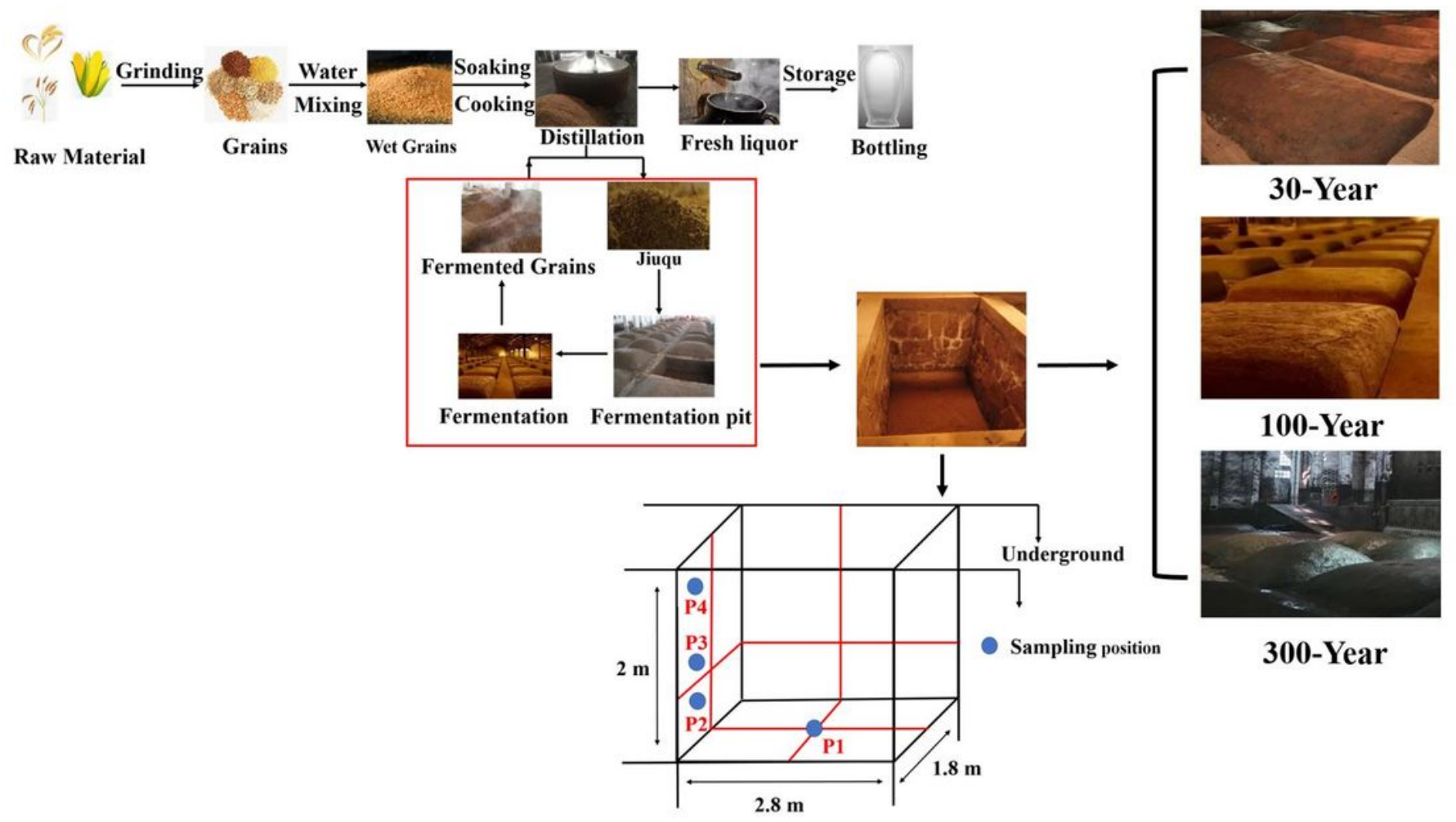

\section{Figure 1}

Traditional production processing of Chinese Strong-Flavoured Liquor (CSFL, the raw materials of CSFL are cereals, mostly sorghum, or a mixture of corn, rice, millet, and wheat; raw material mixtures are anaerobically fermented for 45-90 days or longer in a specialized rectangular soil pit, and we selected the pit cellar that was continuously used for the 30-, 100-, and 300-year cellars as a model to investigate microbial compositions, roles, and metabolic functions) 
A

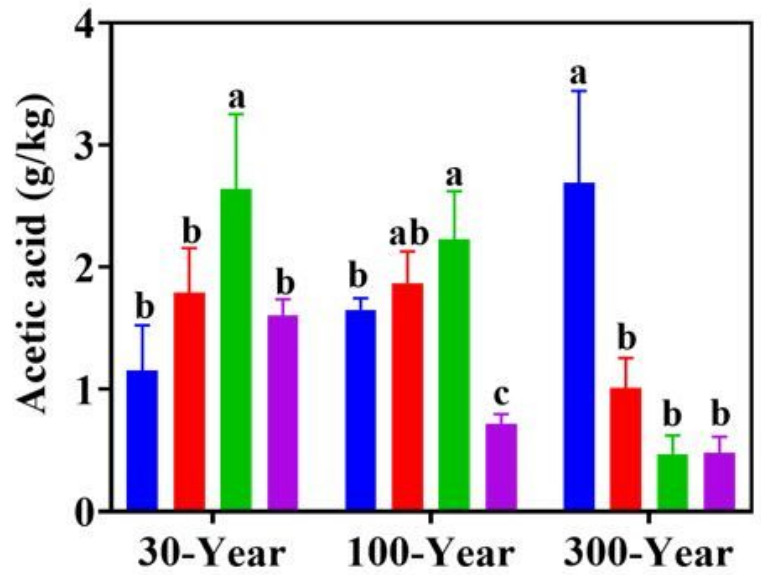

$\mathbf{C}$

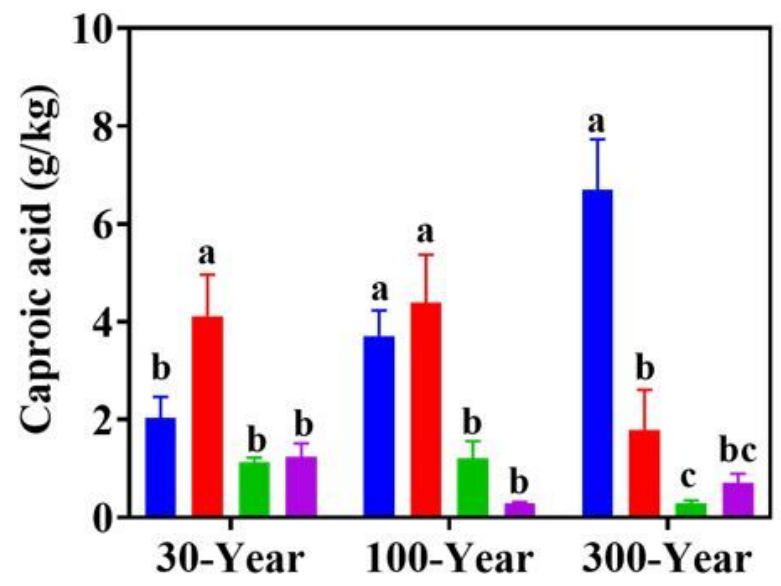

B

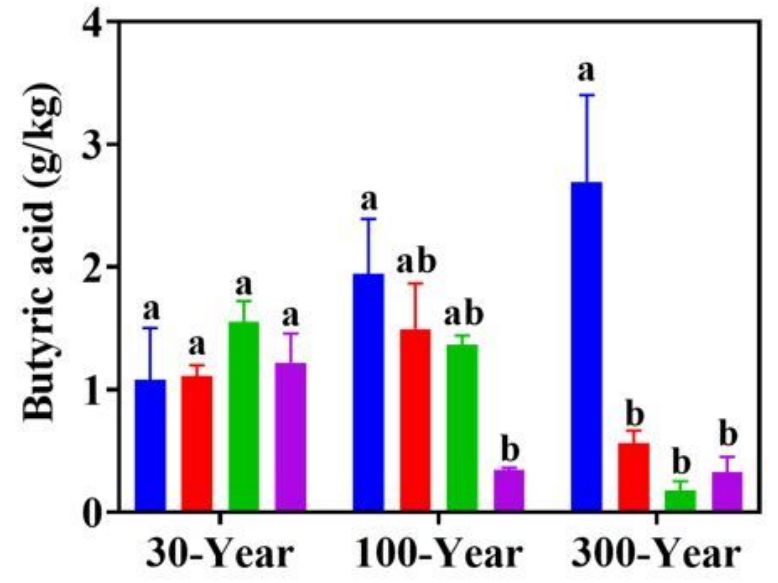

D

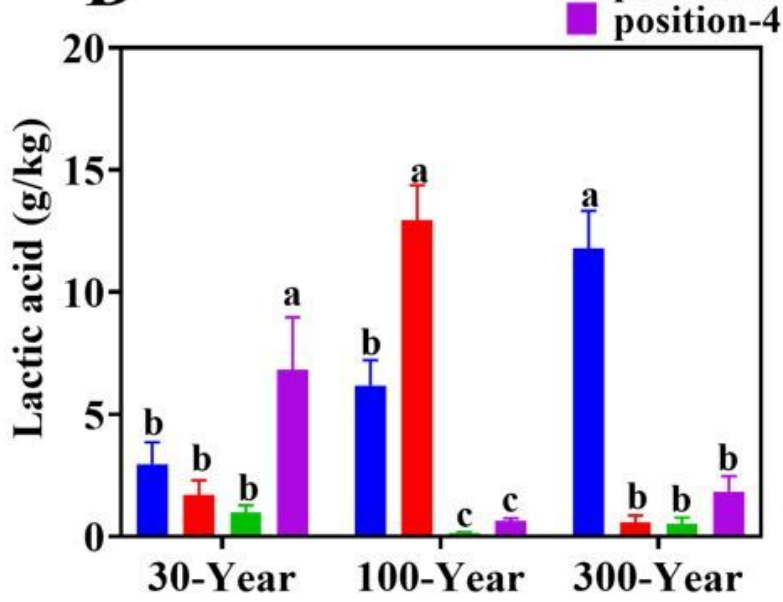

Figure 2

Difference in main organic acid contents in the spatiotemporal niche (the significance analysis refers to the comparison of different spatial locations of the same organic acid in the same year). The content of (A) acetic acid, (B) butyric acid, (C) caproic acid, and (D) lactic acid. 
A
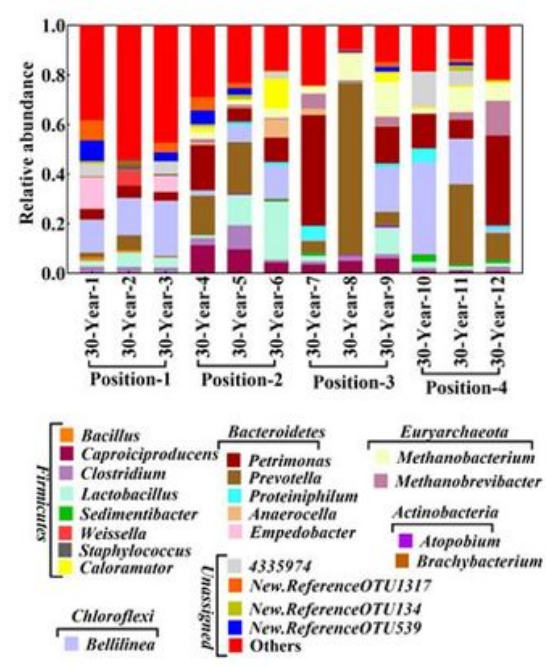

B
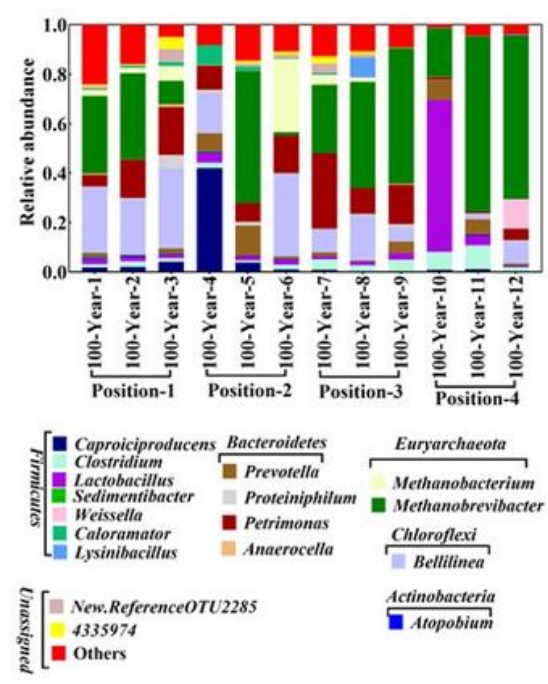

C

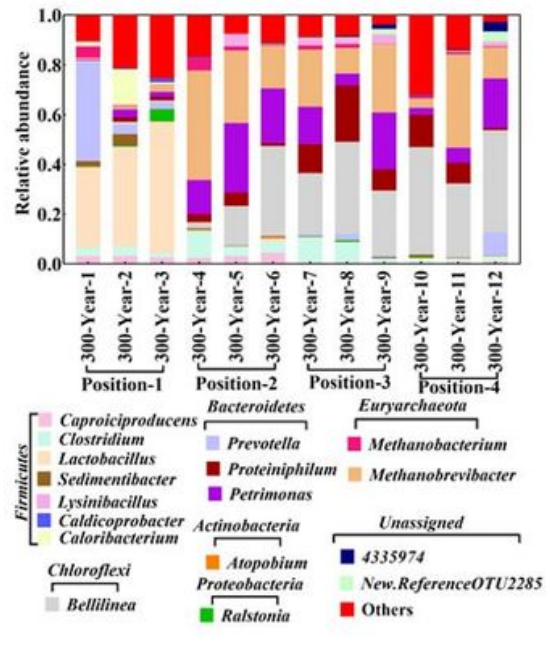

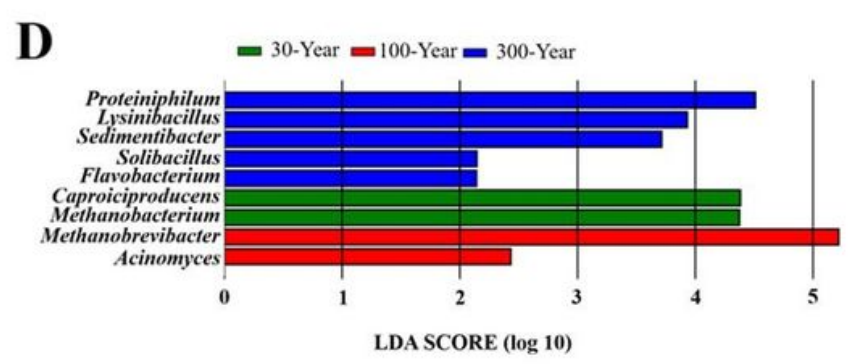

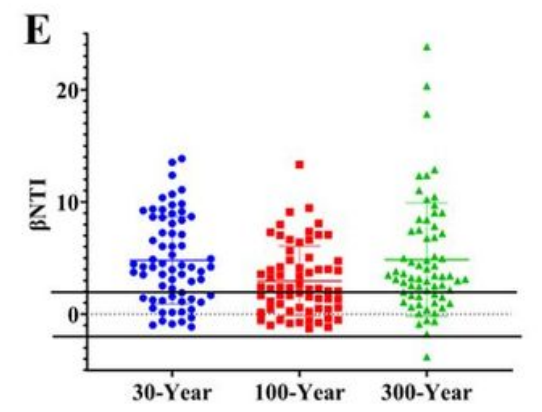

Figure 3

Microbial community composition of PM in different cellars: (A) 30-year-old cellar, (B) 100-year-old cellar, and (C) 300-year-old cellar (D) Linear discriminant effect size (LEfSe) analysis of the bacterial composition in the 30-, 100-, and 300-year-old PM samples (LDA>2 and $p<0.05$ ) (E) Distribution box plots of $\beta N T I$ of bacteria in the 30-, 100-, and 300-year-old PM samples. The horizontal red-dashed lines indicate the upper and lower significance limits of $\beta N T I=+2$ and -2 , respectively. 


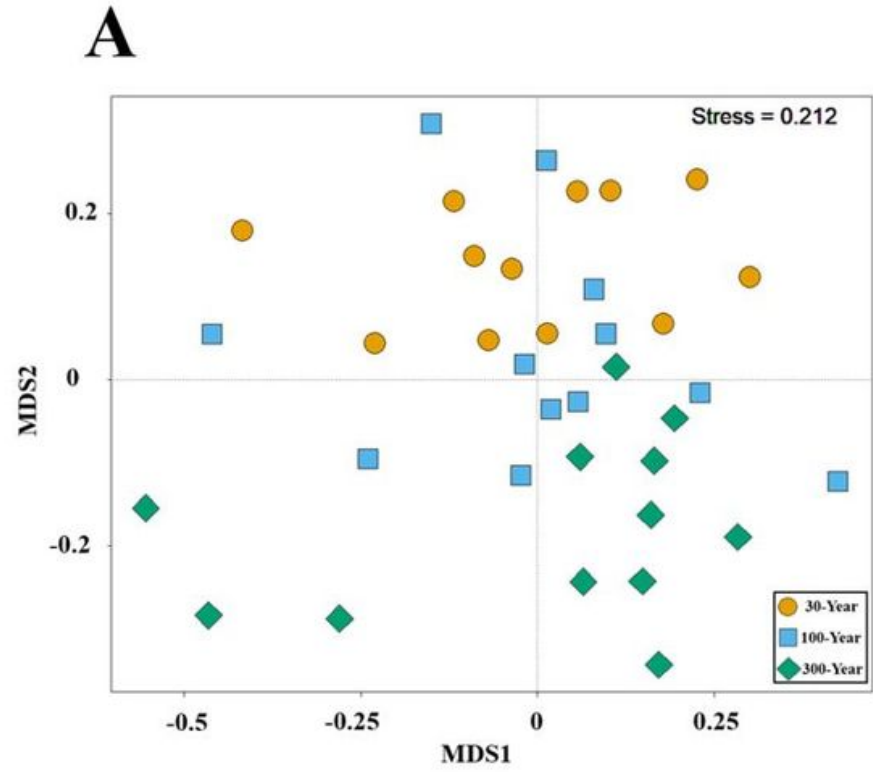

B

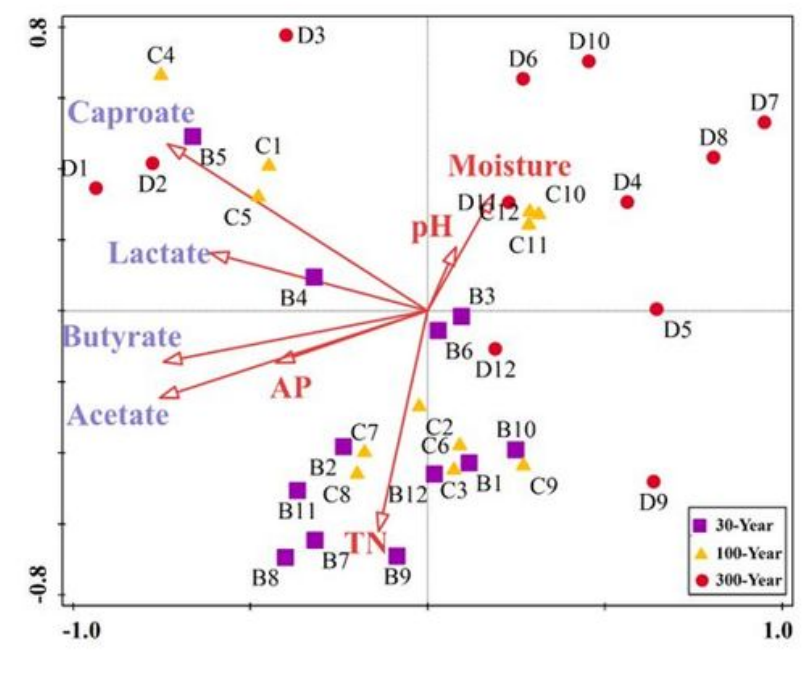

Figure 4

(A) NMDS analysis of bacterial microbiota community in the 30-, 100-, and 300-year-old PM samples. (B) RDA between prokaryotic community structure and environmental parameters in the 30-, 100-, and 300year-old PM samples. 


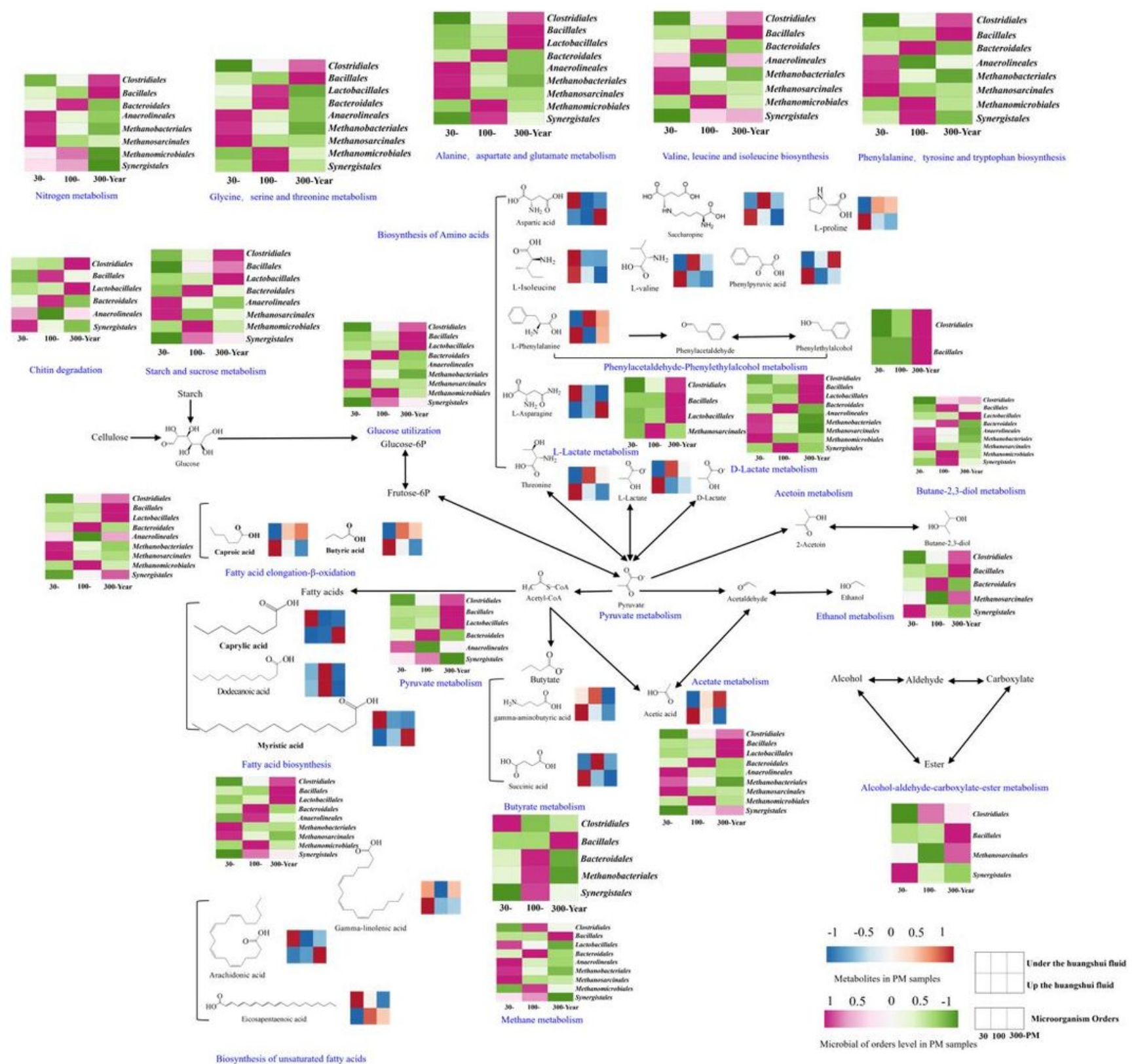

\section{Figure 5}

Schematic presentation of metabolites and microbiota orders related to flavour generation and substrate degradation pathways in the 30-, 100-, and 300-year-old PM samples. The heat maps of all genes annotated to the $135 \mathrm{KEGG}$ enzymes in a predicted metabolic network for substrate degradation and flavour generation in PM, relating to the top 9 orders. The values were transformed with Z-score normalization. Blue tests represent key substrate and flavour metabolic pathways in PM. The blue-red heat map refers to the abundance of metabolites at positions under the huangshui fluid and above the huangshui fluid. The green-purple heat map refers to the abundance of the enzymes related to the substrate and flavour generation pathways. 


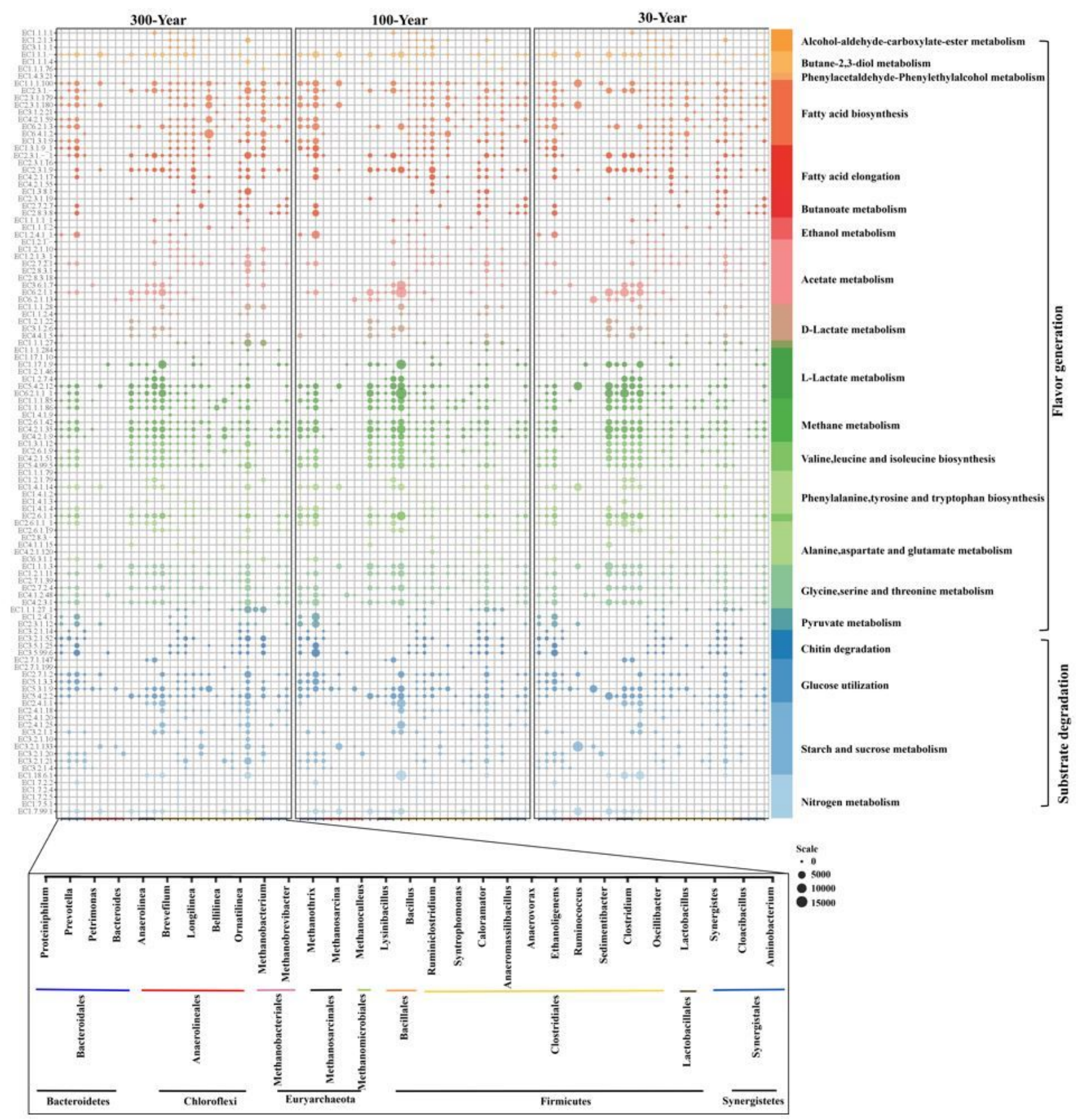

Figure 6

Taxonomic and functional distribution for substrate degradation and flavour generation, relating to the top 30 genera and $135 \mathrm{KEGG}$ enzymes. The diagram is composed of three sub-plots, which correspond to the samples (30-, 100-, and 300-year-old samples). Vertical coordinates indicate the EC numbers of the functional enzymes. Horizontal coordinates, which indicate taxonomic classifications, are magnified at the bottom. Bubble size is proportional to the absolute abundance of the corresponding enzyme-coding genes. Highlighted colour bars help to rapidly locate the corresponding position of specific families (on Xaxis) and functions (on Y-axis). 


\section{Supplementary Files}

This is a list of supplementary files associated with this preprint. Click to download.

- supplymentmaterials.docx 